\title{
LA SUSTITUCIÓN DE TRABAJO POR CAPITAL EN \\ LA INDUSTRIA MANUFACTURERA MEXICANA \\ EN EL PERÍODO 1999-2004
}

\section{REPLACEMENT WORKING CAPITAL IN MEXICAN MANUFACTURING}

IN THE PERIOD 1999-2004

\author{
Miguel Cruz Vásquez $\mid$ Universidad Popular Autónoma del Estado de Puebla \\ Correo: diez4diez@yahoo.com.mx, \\ miguel.cruz@upaep.mx
}

\section{RESUMEN}

El objetivo de este trabajo es analizar la sustitución entre los factores productivos trabajo y capital en la industria manufacturera mexicana en los años 1999 y 2004, segmentando el mercado laboral en sectores formal e informal. Utilizamos la información de las 282 clases industriales que componen el sector manufacturero mexicano provenientes de los Censos Económicos 1999 y 2004 del INEGI. Los resultados obtenidos muestran que las empresas manufactureras sustituyen significativamente capital por trabajadores formales e informales. Adicionalmente se obtuvo evidencia de que el trabajo formal y el trabajo informal son complementarios.

Palabras clave: industria manufacturera, sustitución, trabajo informal, trabajadores, capital.

Clasificación JEL: C13, J23, J38 


\section{ABSTRACT}

The aim of this work is analyze the substitution between two productive factors in the Mexican manufacturing industry between 1999 and 2004: labor and capital, segmenting the labor market into formal and informal sectors. We used information from the 282 industrial classes in the Mexican manufacturing sector described by the 1999 and 2004 INEGI Economic Census. Additionally, the results indicate that Mexican manufacturing firms substitute meaningfully capital for workers in formal and informal sectors. Additionally, empirical evidence was obtained that the workers in formal and informal sectors are complementary or no overlapping. Key words: manufacturing industry, substitution, jobs in the informal sector, employees, capital.

JEL Classification: C13, J23, J38.

\section{INTRODUCCIÓN}

Las regulaciones laborales y los costos extra salariales a menudo son considerados en la literatura responsables de que en los países de América Latina existan una ineficiente asignación del factor trabajo, bajos niveles de productividad, continuo crecimiento del desempleo y del empleo informal y precario, así como sustitución de trabajo por capital 1,

La tasa de desempleo abierto ha crecido constantemente desde los noventa, de 2.8 por ciento en 1992, 3.7 por ciento en 1994, 2.2 por ciento en 2000, 2.7 por ciento en 2002, 3.4 por ciento en 2007, 3.7 por ciento en 2008 y 5.7 por ciento en 2010 (Datos anteriores a 2005 obtenidos de la página: http:/www.ursecon/ecolat/mx/mebbdesem.html, que reporta cifras del INEGI. Los datos de 2005 en adelante se obtuvieron de la Encuesta Nacional de Ocupación y Empleo ENOE 2010, del INEGI). La informalidad actualmente se estima que abarca aproximadamente al 60 por ciento de la población económicamente activa, es decir que incluye a casi 25 millones de mexicanos. http://www.eumed.net/cursecon/ecolat/mx/2005/mcro-informal.htm

Revista de Economía - Vol. XXVIII - Núm. 78 porque se juzga que tanto las regulaciones laborales como loscostos extra salariales, limitan las condiciones para que el trabajador pueda laborar eficientemente. (BID: 2010:29-39). No obstante, dado que coexisten diferentes visiones acerca del impacto de las regulaciones laborales y los costos extra salariales sobre el empleo, es importante la evidencia empírica para orientar las investigaciones sobre la materia.

Por otra parte, de acuerdo con la teoría neoclásica, la demanda de trabajo puede reducirse debido a cuatro razones: a) porque el precio de la producción de la empresa disminuye, lo que ocasiona que disminuya el ingreso del producto marginal que es el valor del producto adicional derivado de cada unidad de trabajo adicional contratada; b) porque el precio de otros factores de producción que son sustitutos del trabajo disminuye, haciendo atractiva la sustitución de éste por aquéllos; c) porque los precios de otros factores de producción que son complementos del trabajo aumentan, disminuyendo el interés por contratar trabajadores; d) porque la incorporación de una nueva tecnología disminuye el producto marginal del trabajo, reduciendo el número de empleos que crea en comparación con los que destruye (por ejemplo, los intercambios electrónicos de llamadas telefónicas son sustitutos de las operadoras telefónicas, pero esa misma tecnología es complemento de los gerentes de los sistemas de programadores e ingenieros electrónicos); aunque los tres últimos motivos se presentan solo en el largo plazo, cuando la empresa ajusta todos sus recursos e incorpora más tecnología a su producción. (Parkin, et al., 2005: 411:413). Una posible explicación a la reducción de la demanda de trabajo en México es que los patrones sustituyen el factor mano de obra por el factor capital debido a los elevados costos extra salariales establecidos en nuestra legislación laboral, que incluyen previsiones laborales como aguinaldos, primas vacacionales, bonificaciones, incentivos y bonos de productividad, asimismo, prestaciones sociales que incluyen las contribuciones patronales a los regímenes de seguridad social (IMSS, ISSSTE, INFONAVIT), las utilidades repartidas a los trabajadores y los pagos por 
indemnización o liquidación del personal 2 , los cuales encarecen en términos relativos la mano de obra respecto al capital, reduciendo la productividad marginal del factor trabajo y restándole competitividad a éste frente al capital. Entre los trabajos publicados que tratan de probar la relación de causalidad de los costos extra salariales sobre la reducción de la demanda de trabajo y su posible desplazamiento por capital para el caso de México y América Latina, se encuentran los de (Montes y Santamaría, 2007:161), (The World Bank, et al., 2004: 29-32) (Lora, 2001: 18), (Lora y Pagés, 1996: 7-10).3

Por ello, en este trabajo planteamos la hipótesis de que en el sector manufacturero mexicano se da la sustitución de trabajadores por capital debido al encarecimiento relativo del trabajo provocados por los costos extra salariales definidos en el párrafo anterior, reduciendo la demanda de trabajadores y aumentando la de capital, para lo cual estimaremos la elasticidad de sustitución entre el trabajo y el capital en el sector mencionado. Es necesario reconocer que los trabajos empíricos que estiman la elasticidad de sustitución exclusivamente para el caso de México son escasos, entre los cuales destaca el realizado por (Ibarra, 1994) que estudia la sustitución capital-trabajo en las maquiladoras manufactureras ubicadas en la frontera con Estados Unidos, mientras que la mayoría de los existentes incluyen a nuestro país dentro de una muestra de varias naciones, para las cuales estiman de manera global la elasticidad de sustitución trabajocapital, como los de (Claro, 2002), (Behrman, 1982) y (Boon, 1973), por lo que la principal aportación de nuestro estudio es tratar de contribuir a la literatura que se ha enfocado a estudiar el tema específicamente para el caso mexicano.

Los costos de despido incluyen actualmente las cantidades monetarias a que tiene derecho el trabajador por despido injustificado de acuerdo con la Ley Federal del Trabajo tales como la indemnización de tres meses de salario, el pago de salarios caídos desde la fecha del despido hasta la de cumplimiento del laudo, y la prima de antiguedad consistente en el pago de 12 dias de salario por cada año de servicios, entre otras.

Por ejemplo, las horas extraordinarias de trabajo en México, junto con Uruguay y Bolivia son pagadas con uno de los mayores recargos en América Latina, el cual fluctua entre un 25 y un 100 porciento adicional. En otro ejemplo, las contribuciones obligatorias a cargo de empresas y trabajadores a diferentes programas de pensiones, ayuda familiar, accidentes de trabajo y desempleo, entre otros, en México, junto con Costes Bolivia y Paraguay son de la más elevadas en el área latinoamericana. (Lora y Pages, 1996: 30-31)
Otra de las aportaciones de nuestra investigación respecto a las que se han publicado anteriormente sobre el tema, es el tipo de función en que nos basamos para la estimación de la elasticidad de sustitución entre el capital y el trabajo, la cual es una función no empleada antes para el caso mexicano, la función de costos translogarítmica con tres insumos (trabajo con prestaciones, trabajo sin prestaciones y capital), que tiene ventajas sobre otras especificaciones debido a su flexibilidad y porque no impone una elasticidad de sustitución constante, si bien el trabajo de (Ibarra, 1994) si se basa en una función translogarítmica pero de producción que incluye tres insumos (trabajo, capital y materiales), mientras que los de (Claro, 2002) y (Boon, 1973) lo hacen sobre una función de producción CES, y el de (Behrman, 1982) sobre una función de producción denominada ACMS (Arrow, Chenery, Minhas y Solow), que es una derivación de la función producción CES, siendo una limitante de esta última la elasticidad de sustitución constante.

Asimismo, nuestras contribuciones incluyen: i) la utilización de una mayor cantidad de datos para estimar la sustitución entre trabajo y capital, ya que empleamos la totalidad de las clases industriales del sector manufacturero mexicano, 282, en tanto que el trabajo de (Ibarra, 1994) solo considera datos de nueve sectores de la industria maquiladora mexicana ubicada en los municipios fronterizos con Estados Unidos, mientras que dentro de los estudios que incluyen a nuestro país dentro de una muestra de un conjunto de países para el cálculo de tal elasticidad, el de (Claro, 2002) incluye datos de 28 ramas industriales manufactureras de 34 países, el de (Behrman, 1982) comprende cifras de 27 sectores manufactureros de 17 países, y el de (Boon, 1973) considera datos de 7 sectores industriales para 21 países; ii) la utilización de cifras más recientes que los estudios empíricos publicados, ya que su base estadística son las cifras de los Censos Económicos 1999 y 2004, mientras que el de (Ibarra, 1994) se basa en series de tiempo anuales para el período 1981-1989, y los de (Claro, 2002), (Behrman, 1982) y (Boon, 1973), utilizan un solo corte transversal, para 1990, 1970 y 1965 respectivamente; iii) la utilización de un procedimiento de estimación econométrica distinto a los empleados en 
trabajos anteriores, el de pooling o combinación de cortes transversales, ya que (Ibarra, 1994) utiliza el procedimiento ISUR (Iterative Seemingly Unrelated Regressions) y los trabajos de (Claro, 2002), (Behrman, 1982) y (Bon, 1973), aplican el procedimiento de Corte Transversal.

Para probar nuestra hipótesis, estimamos econométricamente las elasticidades precio cruzadas y las elasticidades de sustitución existentes entre el trabajo formal y el trabajo informal, entre el capital y el trabajo formal, y entre el capital y el trabajo informal. Además estimamos como base de las anteriores las elasticidades precio propias de los siguientes factores productivos: trabajo formal, trabajo informal y capital. En este trabajo suponemos que el trabajo formal es aquel que cuenta con las prestaciones extrasalariales ya mencionadas antes; que el trabajo informal es aquel que solo recibe honorarios, propinas, igualas y comisiones como remuneración, y no recibe las prestaciones extrasalariales ya expuestas; y que el capital es tanto la cantidad de capital nuevo adquirido por las empresas como la cantidad de capital repuesto debido a la depreciación. Los datos utilizados en este estudio se obtuvieron de los Censos Industriales de 1999 y 2004 levantados por el Instituto Nacional de Estadística, Geografía e Informática (INEGI), 4 correspondientes a un total de 282 clases industriales que componen el sector manufacturero mexicano, por lo que se tienen dos cortes transversales para dichos años.

El método de estimación que utilizamos es el de combinación de cortes transversales independientes 5 , llamado también de datos pooled.

4"La comparabilidad de los censos industriales 1999 y 2004 a nivel clase es posible debido a que los resultados de los censos económicos de 1999 fueron dados a conocer por única vez y para facilitar a los usuarios el paso hacia la nueva clasificación 1999 fueron la óntica de dos clasificadores: a) El Sistema Clasificación Industrial de América del Norte (SCIAN), clasificador oficial de actividades económicas desde 1997; y b) la Clasificación Mexicana de Actividades y Productos (CMAP) de 1994, clasificación que venía empleándose desde 1981." Se pudieron comparar ambos censos a nivel de clase industrial, ya que ambos están codificados siguiendo el clasificador SCIAN. (INEGI, 2004:7), (INEGI, 2004:3).

En este procedimiento de estimación por mínimos cuadrados, suponemos que el error en cada ecuación del sistema no está correlacionado con la variables explicativas en cada una de las ecuaciones, es decir que no hay problemas de endogeneidad en cada una de las ecuaciones que constituyen el sistema porque las variables independientes, en este caso los precios de los factores, son variables exogenas. (Ver Woolridge, 2001: 408) Esto es coherente si suponemos que en cada una de las empresas manufactureras, los salarios son fijados exógenamente de acuerdo con las negociaciones salariales entre ellas y sus trabajadores.

Revista de Economía - Vol. XXVIII - Núm. 78
El trabajo se organiza de la siguiente manera: en la segunda sección se presenta el marco teórico, en la tercera sección se muestra una revisión de la bibliografía, en la cuarta se expone la metodología; en la quinta sección se muestran los resultados; y en la sexta se concluye.

\section{MARCO TEÓRICO}

Aunque el grado de sustitución de un factor por otro se puede medir de manera natural a través de la elasticidad cruzada de la demanda de factores 6 ; sin embargo, por razones históricas, la mayoría de los economistas no utilizan esta elasticidad cruzada, sino una medida relacionada con ella, conocida como la elasticidad parcial de sustitución, también denominada elasticidad de sustitución Hicks-Allen, concebida para medir la facilidad de sustituir un insumo por otro manteniendo el producto y los precios de los otros insumos constantes. (Nicholson, 1997: 255), (Hamermesh, 1993: 33:35).

Por ejemplo, $\sigma_{\mathrm{ij}}$, la elasticidad parcial de sustitución entre dos factores $\mathrm{x}_{\mathrm{i}} \mathrm{y}_{\mathrm{j}}$, se define como la variación en la proporción utilizada de los dos factores $\underline{x i}$ en respuesta a una variación de los precios de dichos

factores, $\stackrel{P j}{-}$, es decir, que queremos calcular la derivada $\frac{\partial \underline{x i}}{\partial \underline{P j}}$ ) . Para el

Por ejemplo, $n_{j}$ es la elasticidad precio crzzada de la demanda del factor i, es decir de x, respecto al precio del factor j, es decir, $\mathrm{P}$, , se calcula mediante el cociente $\frac{d \underline{d P})}{\left(\frac{d P}{)}\right.}$, manteniendo otras cosas constantes. (Nicholson, W., 1997: 255), (Hamermesh, 1993: 33:35).

Enero a Junio de 2012 - Págs: 37-79 
caso de los factores capital $(\mathrm{K})$ y trabajo $(\mathrm{L}), \sigma_{\mathrm{ij}}$, la elasticidad parcial de sustitución entre los factores capital y trabajo sería la variación en la proporción utilizada de los factores $\frac{K}{-}$, en respuesta a una variación los precios de dichos factores $\stackrel{w}{-}$, donde $\mathrm{w}$ es el salario o precio del trabajo y (r) el rendimiento del capital, manteniendo constante Y, es decir, la escala de producción. En otras palabras, queremos calcular la derivada $\frac{\partial \underline{K})}{\partial \underline{w}-)}$ a lo largo de una isocuanta, que es una curva que muestra las combinaciones de $\mathrm{K}$ y L que pueden generar el mismo nivel de producción. (Nicholson, 1997, p. 206)

La elasticidad parcial de sustitución entre distintos insumos, por ejemplo entre diferentes tipos de trabajo, permite la utilización de la teoría de la producción con varios insumos para inferir cómo cambios en la tasa salarial de un grupo de trabajadores afecta no sólo la demanda de este grupo de trabajadores, sino también la demanda de otros insumos y la demanda de trabajo de otros grupos de trabajadores.

El enfoque de la demanda de trabajo utilizado en este estudio supone que los salarios son establecidos exógenamente 7; porque consideramos que la oferta de trabajo es perfectamente elástica ya sea porque hay suficiente desempleo o porque es pagado un salario mínimo efectivo a los trabajadores, por lo que el equilibrio en el mercado de trabajo es determinado solo por la demanda. Este enfoque nos permite calcular los impactos de cambios exógenos en el precio del trabajo, por ejemplo de cambios de los costos extra salariales sobre la demanda de trabajo que, cuenta con prestaciones extra salariales, asimismo sobre la demanda de trabajo que no cuenta con prestaciones extra salariales 8 y también sobre la demanda de capital.

Este enfoque de estudio de la demanda de trabajo contrasta con otro enfoque del estudio de la demanda de trabajo en el cual el nivel de empleo es determinado sólo por la oferta de trabajo perfectamente inelástica en la a demanda de trabajo determina el salario pagado a los trabajadores. (Hamermesh, 1993:18-20)
Para ilustrar la utilización de este enfoque en la estimación del impacto de la reducción de los costos salariales sobre la demanda de trabajo, podemos representar en la figura 1 una reducción en el salario de $\mathrm{W}^{0}$ a $\mathrm{W}^{1}$ que ocasiona un desplazamiento de la oferta de trabajo de $\mathrm{S}^{0}$ a $\mathrm{S}^{1}$. el cual produce un aumento en el empleo de $\mathrm{L}^{0}$ a $\mathrm{L}^{1}$. En este caso, el efecto directo del shock recae en los salarios (W), y esto a su vez produce un aumento de la cantidad demandada de empleo (E), que puede inferirse si conocemos la pendiente de la curva de la demanda de trabajo $\mathrm{AC} / \mathrm{CB}$, o la elasticidad precio propia de la demanda de trabajo, la que a su vez se calcula a través de la fórmula siguiente $\frac{\left(\frac{d L}{\mathrm{~L}}\right)}{\left(\frac{d W}{W}\right)}$, manteniendo otras cosas constantes. (Nicholson, 1997: 255).

Figura 1.

Oferta de trabajo infinitamente elástica

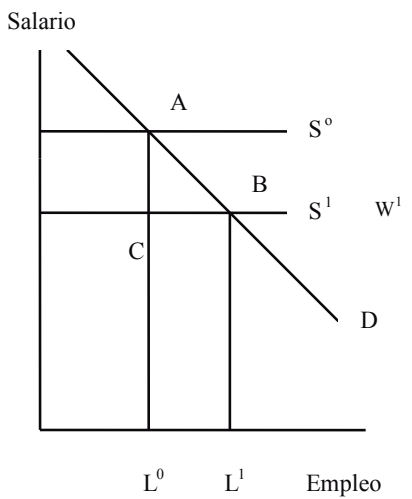

Fuente: Hamemesh, 1993:18

${ }^{8}$ Tal enfoque es congruente con la manera en que se establecen los salarios y las prestaciones extrasalariales las empresas manufactureras mexicanas, si suponemos que en cada una de ellas los salarios son fijados exógenamente de acuerdo con las negociaciones salariales efectuadas con sus trabajadores. 
Para la estimación de las elasticidades de la demanda del trabajo antes mencionadas, en este trabajo utilizamos la función de Costos Translogarítmica 9 , debido a la flexibilidad de ésta y a que su estimación no impone restricciones sobre los valores estimados de las elasticidades, misma que para $\mathrm{n}$ insumos es mostrada en la ecuación (1) (Hamermesh, 1993:31)

$$
\text { (1) } \ln C=\ln a_{0}+\sum_{i=1}^{n} a_{i} \ln w_{i}+\frac{1}{2} \cdot \sum_{i=1}^{n} \sum_{j=1}^{n} b_{i j} l n w_{i} \ln w_{j}+a_{Y} \ln Y+\frac{1}{2} b_{Y Y}(\ln Y)^{2}+\sum_{i=1}^{n} b_{i Y} \ln w_{i} \ln Y
$$

Donde $b_{i j}=b_{j i}$. Para que una función de costos cumpla con los requisitos que establece la teoría debe, entre otras cosas, ser homogénea de grado 1 en precios 10 , dado Yo nivel de producto, lo que implica las siguientes restricciones sobre la ecuación (1):

$$
\text { (2) } \sum_{i=1}^{n} a_{i}=1 ; b_{i j}=b_{j i} ; \sum_{i=1}^{n} b_{i j}=0 \text {, para toda } j \text {. }
$$

La primera y tercera igualdades en la ecuación anterior resultan de los supuestos de que $\mathrm{C}$ es lineal homogénea en los $\mathrm{w}$ i (incrementos proporcionales en los $\mathrm{w}_{\mathrm{i}}$ elevan proporcionalmente los costos), y de que es necesario y suficiente que $\mathrm{b}_{\mathrm{iy}}=0 \forall \mathrm{i}=1, \ldots$. para que la funció de costos translog sea homotética ${ }^{11}$. El segundo supuesto surge del requisito de que la función de costos sea simétrica tal que que $b_{i j}=b_{j i}$.

Se podría estimar directamente la función de costos translogarítmica (1), pero es más conveniente la estimación de ecuaciones de demanda óptimas minimizadoras de costos, transformadas en ecuaciones de participaciones de costos (no insumo-producto), lineales en los parámetros, de la forma.

${ }^{9}$ Las funciones de costos translogarítmicas son aproximaciones de series de Taylor de segundo orden en logaritmos para funciones de costos arbitrarias, muy generales, en las que las relaciones de las demandas de insumos minimizadoras de costos dependen del nivel de producto. (Hamermesh, 1993:31)

10 Una función de costos homogenea de grado uno, también denominada linealmente homogénea o de rendimientos constantes a escala implica que cuando todos los insumos se duplican o varian en cualquier otra proporción, los costos también se duplican o varian en esa otra proporción (Nicholson, 1997: 233).

La condición de homoteticidad implica que la razón de insumos demandados es independiente de la escala de producción a cada uno de las razones de precios de los factores, es decir, que la relación marginal de sustitución entre los factores solo depende de las cantidades relativas de los mismos y no de sus cantidades absolutas.

\section{(3) $\frac{\partial \ln \mathrm{C}}{\partial \ln \mathrm{w}_{\mathrm{i}}}=\frac{\mathrm{w}_{\mathrm{i}}}{\mathrm{C}} \cdot \frac{\partial C}{\partial \mathrm{w}_{\mathrm{i}}}=\frac{\mathrm{w}_{\mathrm{i}} \mathrm{X}_{\mathrm{i}}}{\mathrm{C}}=\mathrm{S}_{\mathrm{i}}=\mathrm{a}_{\mathrm{i}}+$ \\ $\sum_{j=1}^{n} b_{i j} \ln w_{j}+b_{i Y} \ln Y, \quad i=1, \ldots \ldots . . n$}

donde $\sum_{\mathrm{i}=1}^{\mathrm{n}} \mathrm{w}_{\mathrm{i}} \mathrm{X}_{\mathrm{i}}=\mathrm{C}$, es decir que la suma ponderada de las cantidades de factores utilizadas, multiplicadas por los precios de los propios factores, nos da el total de costos. Definiendo las participaciones de costos de los factores $S_{i} \equiv \frac{w_{i} X_{i}}{C}$, se sigue que

$$
\sum_{\mathrm{i}=1}^{\mathrm{n}} \mathrm{S}_{\mathrm{i}}=1
$$

Esta condición aditiva del sistema de ecuaciones de participaciones (3) tiene importantes implicaciones para la estimación econométrica. Por ejemplo, para una función de costos de cuatro insumos (capital K, trabajo $\mathrm{L}$, energía $\mathrm{E}$ y materiales $\mathrm{M}$ ), que tiene la forma translog, las ecuaciones de participación de costos tendrán la forma siguiente. (Berndt, 1991 460-461) (Hamermesh, 1993: 39-40). (Berndt y Wood, 1975: 259-268)

(4) $\mathrm{S}_{\mathrm{K}}=\mathrm{a}_{\mathrm{K}}+\mathrm{b}_{\mathrm{KK}} \ln \mathrm{W}_{\mathrm{K}}+\mathrm{b}_{\mathrm{KL}} \ln \mathrm{W}_{\mathrm{L}}+\mathrm{b}_{\mathrm{KE}} \ln \mathrm{W}_{\mathrm{E}}+\mathrm{b}_{\mathrm{KM}} \ln \mathrm{W}_{\mathrm{M}}+\mathrm{b}_{\mathrm{KY}} \ln \mathrm{Y}$

$$
\begin{aligned}
& S_{\mathrm{L}}=\mathrm{a}_{\mathrm{L}}+\mathrm{b}_{\mathrm{LK}} \ln \mathrm{W}_{\mathrm{K}}+\mathrm{b}_{\mathrm{LL}} \ln \mathrm{W}_{\mathrm{L}}+\mathrm{b}_{\mathrm{LE}} \ln \mathrm{W}_{\mathrm{E}}+\mathrm{b}_{\mathrm{LM}} \ln \mathrm{W}_{\mathrm{M}}+\mathrm{b}_{\mathrm{LY}} \ln \mathrm{Y} \\
& \mathrm{S}_{\mathrm{E}}=\mathrm{a}_{\mathrm{E}}+\mathrm{b}_{\mathrm{EK}} \ln \mathrm{W}_{\mathrm{K}}+\mathrm{b}_{\mathrm{EL}} \ln \mathrm{W}_{\mathrm{L}}+\mathrm{b}_{\mathrm{EE}} \ln \mathrm{W}_{\mathrm{E}}+\mathrm{b}_{\mathrm{EM}} \ln \mathrm{W}_{\mathrm{M}}+\mathrm{b}_{\mathrm{EY}} \ln \mathrm{Y} \\
& \mathrm{S}_{\mathrm{M}}=\mathrm{a}_{\mathrm{M}}+\mathrm{b}_{\mathrm{MK}} \ln \mathrm{W}_{\mathrm{K}}+\mathrm{b}_{\mathrm{ML}} \ln \mathrm{W}_{\mathrm{L}}+\mathrm{b}_{\mathrm{ME}} \ln \mathrm{W}_{\mathrm{E}}+\mathrm{b}_{\mathrm{MM}} \ln \mathrm{W}_{\mathrm{M}}+\mathrm{b}_{\mathrm{MY}} \ln \mathrm{Y}
\end{aligned}
$$


En este sistema, las elasticidades parciales de sustituci ón ${ }^{12}$ son:

$$
\sigma_{i j}=\frac{\left(b_{i j}+S_{i} S_{j}\right)}{S_{i} S_{j}}
$$$$
\mathrm{i}, \mathrm{j}=1, \ldots \mathrm{n} \text {, }
$$

pero $\mathrm{i} \neq \mathrm{j}$;

$\mathrm{y}$

$$
\sigma_{i i}=\frac{\left(b_{i i}+S_{i}^{2}-S_{i}\right)}{S_{i}^{2}}, \quad \quad i=1, \ldots . . n \quad \text { pero } i \neq j
$$

Donde $\mathrm{S}_{\mathrm{i}}=\mathrm{w}_{\mathrm{i}} \mathrm{X} / \mathrm{C}$; mientras que las elasticidades de la demanda no condicionada, son ${ }^{13}$

$$
\eta_{i j}=\frac{b_{i j}+S_{i} S_{j}}{S_{i}}, \quad i, j=1, \ldots \ldots, n, \quad \text { pero } i \neq j
$$

$$
\eta_{\mathrm{ii}}=\frac{\left(\mathrm{b}_{\mathrm{ii}}+\mathrm{S}_{\mathrm{i}}^{2}-\mathrm{S}_{\mathrm{i}}\right)}{\mathrm{S}_{\mathrm{i}}}, \quad \mathrm{i}=1, \ldots \ldots, \mathrm{n}
$$

La estimación individual o simultánea del sistema de ecuaciones (3), sujeta a las restricciones que se mencionaron, proporciona los coeficientes necesarios para estimar las elasticidades de sustitución y las elasticidades precio directas/cruzadas mostradas en las ecuaciones (5) a (8).

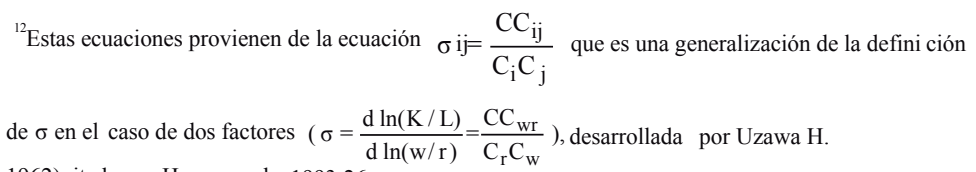

Como se ha puesto en evidencia, para estimar la elasticidad de la demanda de trabajo respecto al salario o a los costos extrasalariales y de ahí la elasticidad cruzada y la elasticidad de sustitución entre los tipos de trabajo y entre los tipos de trabajo y el capital, necesitamos conocer datos característicos de las empresas comos los niveles de empleo, los salarios, la demanda de sus productos, y algunos de los precios y cantidades de sus demás insumos.

Con objeto de reflejar la segmentación del mercado laboral mexicano en los sectores formal e informal, incluiremos en esta función de costos los siguientes factores productivos: el trabajo con prestaciones extrasalariales (trabajadores con derecho a prestaciones sociales, reparto de utilidades e indemnizaciones por despido) L1; el trabajo sin prestaciones extrasalariales (trabajadores sin derecho a prestaciones sociales, reparto de utilidades e indemnizaciones por despido) L2, es decir, el trabajo por honorarios; y el capital $\mathrm{K}$, mismos que tienen respectivamente los siguientes precios: w1, w2, y wK.

\section{REVISIÓN DE ESTUDIOS EMPÍRICOS}

Como ya comentamos en la introducción, escasean los trabajos empíricos realizados exclusivamente para la estimación de la sustitución de trabajo por capital en nuestro país y la mayoría de los que se refieren a nuestro país, lo hacen marginalmente al incluirlo dentro de una muestra de naciones para las que se hace la estimación de una manera global.

Uno de los trabajos más importantes elaborados para México, es el de (Ibarra, 1994), que estimó las elasticidades precio y las elasticidades parciales de sustitución Hicks-Allen entre los insumos trabajo, capital y materiales para la industria maquiladora ubicada en los municipios fronterizos de México 
materiales para la industria maquiladora ubicada en los municipios fronterizos de México con Estados Unidos, basándose en una Función de Producción Translogarítmica que incluye estos tres insumos, utilizando datos de corte transversal de 9 sectores económicos y de series de tiempo anuales para el período 1981-1989, y el procedimiento de estimación ISUR (Iterative Unseemingly Unrelated Regression). Para ello midió los costos laborales sumando los gastos en sueldos, salarios y compensaciones; los costos de materiales sumando los gastos en materias primas, empacado y envasado; y los costos de capital con la suma de los gastos en renta de maquinaria y equipo, renta de edificios y terrenos, energía, teléfonos, telégrafo, télex, impuestos, fletes, mantenimiento de maquinaria y edificios. Sus estimaciones de elasticidades de sustitución fueron de -1.96 entre trabajo y capital, de 2.59 entre trabajo y materiales y de 2.93 entre capital y materiales, por lo que el trabajo y el capital son complementos y los materiales son sustitutos del trabajo y el capital, y dado que la elasticidad de sustitución entre el capital y los materiales es mayor que la elasticidad de sustitución entre el trabajo y los materiales, los materiales son más sustitutos del capital que del trabajo. Sus estimaciones de las elasticidades precio de los factores trabajo, capital y materiales fueron $-1.74,-1.96$ y -0.68 respectivamente, por lo que el trabajo y el capital son relativamente elásticos en su demanda, mientras que los materiales son inelásticos. Sus estimaciones de las elasticidades del trabajo y capital respecto al precio de los materiales fueron 1.95 y 2.20 respectivamente, por lo que el trabajo y el capital son muy elásticos al precio de los materiales, mientras que las elasticidades de los materiales a los precios del trabajo y el capital fueron 0.34 en ambos casos, por lo que los materiales son poco sensibles a cambios en los precios del capital y el trabajo. Entre los trabajos empíricos realizados para un conjunto de países que incluyen a México, se encuentra el de (Claro, 2002), que estimó las elasticidades de sustitución entre capital y trabajo para firmas que operan en mercados perfectamente competitivos basándose en funciones de producción CES que tienen rendimientos constantes a escala, aplicadas a una muestra de 34 países que incluyen 28 industrias manufactureras utilizando la estima- ción de corte transversal con datos de 1990. Los datos utilizados son el número de trabajadores, el valor agregado nominal y el pago nominal de salarios y se obtuvieron de la Base de Datos Estadística denominada UNIDO (United Nations Industrial Development Organization), que contiene datos del período $1963-1996$ para 180 países. La elasticidad de sustitución estimada para cada industria, excepto en cinco de ellas (bebidas, tabaco, refinación de petróleo y maquinaria no eléctrica, hierro y acero y maquinaria no eléctrica) es mayor a 0.7 con lo que la hipótesis nula de Cobb-Douglas de que la elasticidad de sustitución es unitaria es rechazada en la mayoría de los casos y se concluye que un aumento del costo relativo del trabajo hace que la firma utilice relativamente más capital que antes, a cualquier escala de producción. Otro de los trabajos de este tipo es el de (Behrman, 1982), en el que se estima la elasticidad de sustitución entre trabajo y capital para un corte transversal de datos promedio del período 1967-1973, que incluye 17 países, entre ellos México, y más de 27 sectores manufactureros con base en el modelo (Arrow, Chenery, Minhas y Solow, 1961), conocido como modelo ACMS y que se deriva de la función CES. Los datos utilizados son el número de empleados, el número de establecimientos, e incluye variables dummy y proxis para representar las características de los países, con el fin de controlar las variables omitidas, asimismo, combina países y sectores para construir una base de datos de 1,723 observaciones, provenientes del documento United Nations, 1976, Yearbook of Industrial Statistics. New York: United Nations. La elasticidad de sustitución entre el trabajo y el capital estimada es de 0.94 estableciendo el capital como variable dependiente y de 1.43 estableciendo el trabajo como variable dependiente, aunque el segundo valor se encuentra sesgado hacia arriba debido al sesgo de simultaneidad, que se presenta si el salario es establecido simultáneamente con el nivel de empleo ${ }_{14}$.

14 Sesgo de simultaneidad es el sesgo que surge al estimar con el procedimiento de Mínimos Cuadrados Ordinarios una ecuación que no debe estimarse sola porque es parte de un sistema de ecuaciones simultáneas, y es una forma importante de ente con la variable dependiente, por lo regular mediante un mecanismo de equilibrio. (Woolridge, 2001: 797)

2012 - Págs: 37-79 
Hacia el interior de los sectores considerados, la elasticidad de sustitución fluctúa entre 0.86 y 1.00. Concluye que la elasticidad de sustitución es cercana en valor a 1.00 , por lo que concuerda con la función CobbDouglas.

También el estudio de (Boon, 1973) es otro de los que incluyen a México dentro de su muestra de países, en el cual se estima la elasticidad de sustitución entre el trabajo y el capital con base en una función producción CES (Función de Elasticidad de Sustitución Constante), aplicada a una muestra de 21 países que constituyen un corte transversal para 1965 e incluye tres sectores clave de la economía mexicana (manufacturas, construcción y minería), dividiendo a su vez al sector manufacturero en 7 subsectores según el esquema de agregación de la matriz insumoproducto.

Mide el capital mediante el valor agregado bruto y el trabajo por el número de personas ocupadas. Las elasticidades de sustitución estimadas fueron de $0.758,0.803$ y 0.875 para los siguientes sectores de la industria ligera: alimentos, bebidas y tabaco, textiles; vestuario e industria del acero; y madera, papel e industria editorial; asimismo, de 0.912, 0.929, 0.802 y 0.848 para los siguientes sectores de la industria pesada: industria química, petróleo y sus derivados e industria del caucho; productos minerales no metálicos; industria de metales básicos; industria de productos metálicos básicos y otras industrias manufactureras.

Explica que en la industria ligera es menor la sustitución de capital por trabajo que en la industria pesada, debido a que esta última es más sensible a los cambios en los precios de los factores porque puede aumentar la intensidad del trabajo implantando turnos múltiples de trabajo, pero que el trabajo calificado en la industria pesada aún en los países de bajo desarrollo, podría ser relativamente caro y tener una oferta inelástica, lo que podría inducir a una sustitución de capital por trabajo.

En lo que concierne a los estudios realizados sobre el tema en otros países, existe una gran cantidad de estimaciones de la elasticidad de sustitución entre el trabajo y el capital realizadas especialmente para el caso de Estados Unidos y otras naciones, muchas de las cuales son clasificadas y se presentan en los cuadros 3.1 y 3.3 de (Hamermesh, 1993) de acuerdo con el nivel de agregación de los datos, encontrándose en el primer cuadro las estimaciones que utilizan datos agregados o de grandes industrias y en el segundo estimaciones que se basan en datos desagregados a nivel de empresa o de pequeña industria.

Dichas estimaciones se reproducen en los cuadros 1 y 2 de este trabajo. En la primera columna de estos cuadros aparece el nombre del autor de la estimación, en la segunda la dimensión seleccionada para la estimación que puede ser corte transversal o series de tiempo, la unidad de observación que puede ser establecimiento ú hogar, la función estimada que puede ser la de producción o la de costos, y el país al que corresponde la estimación, que puede ser Estados Unidos u otros países.

En la tercera columna se presentan el tipo de trabajo utilizado en la estimación, la periodicidad de las cifras utilizadas, el período de medición y el procedimiento de estimación de la elasticidad de sustitución entre el capital y el trabajo.

En la cuarta columna se muestra el valor de la elasticidad de sustitución estimada.

Cuadro 1. Estimaciones de $\sigma$ utilizando datos agregados o de grandes industrias

\begin{tabular}{|c|c|c|c|}
\hline Estudio & Categoría & Descripción & \\
\hline Dhrymes (1969) & $\begin{array}{l}\text { Series de Tiempo, } \\
\text { establecimiento, estimación } \\
\text { directa de función de costos o } \\
\text { de producción, E.U. }\end{array}$ & $\begin{array}{r}\text { Horas totales privadas, } \\
\text { trimestralmente, } 1948-60 ; \\
\text { condición de producto marginal }\end{array}$ & 0.75 \\
\hline Hamermesh (1983) & $\begin{array}{l}\text { Series de Tiempo, } \\
\text { establecimiento, estimación } \\
\text { directa de función de costos o } \\
\text { de producción E.U. }\end{array}$ & $\begin{array}{l}\text { No agrícola privado, } \\
\text { trimestralmente1955-78; condición } \\
\text { de producto marginal, atención a la } \\
\text { medición de los costos laborales }\end{array}$ & 0.47 \\
\hline $\begin{array}{l}\text { Drazen, Hamermesh, y } \\
\text { Obst (1984) }\end{array}$ & $\begin{array}{l}\text { Serie de Tiempo, } \\
\text { establecimiento, estimación } \\
\text { directa de función de costos o } \\
\text { de producción otros países }\end{array}$ & $\begin{array}{r}\text { Horas-trabajador manufacturero, } \\
\text { trimestralmente, } 10 \text { países OCDE, } \\
\text { principalmente } 1961-80 \text {; condición } \\
\text { de producto marginal. }\end{array}$ & 0.21 \\
\hline $\begin{array}{lll}\text { Lucas } & \text { y } & \text { Rapping } \\
(1970) & & \end{array}$ & $\begin{array}{l}\text { Serie de Tiempo, } \\
\text { establecimiento, estimación } \\
\text { directa de función de costos o } \\
\text { de producción E.U. }\end{array}$ & $\begin{array}{l}\text { Producción agregada-horas } \\
\text { trabajador, anual, 1930-65; } \\
\text { condición producto marginal, } \\
\text { sistema oferta-demanda. }\end{array}$ & 1.09 \\
\hline
\end{tabular}




\begin{tabular}{|c|c|c|c|}
\hline $\begin{array}{lll}\text { Black } & \text { y } & \text { Kelejian } \\
(1970) & & \end{array}$ & $\begin{array}{l}\text { Serie de Tiempo, } \\
\text { establecimiento, estimación } \\
\text { directa de función de costos o } \\
\text { de producción, E.U. }\end{array}$ & $\begin{array}{l}\text { Horas-trabajador privado no } \\
\text { agrícola, trimestralmente, } 1948-65 \\
\text { condición producto marginal, } \\
\text { sistema oferta-demanda. }\end{array}$ & 0.36 \\
\hline Liu y Hwa (1974) & $\begin{array}{l}\text { Serie de Tiempo, } \\
\text { establecimiento, estimación } \\
\text { directa de función de costos o } \\
\text { de producción, E.U, }\end{array}$ & $\begin{array}{r}\text { Horas-trabajador privado, } \\
\text { mensualmente, 1961-71, condición } \\
\text { de producto marginal, sistema } \\
\text { oferta-demanda }\end{array}$ & 0.67 \\
\hline $\begin{array}{lll}\begin{array}{l}\text { Beach } \\
(1983)\end{array} & \text { y } & \text { Balfour } \\
\end{array}$ & $\begin{array}{l}\text { Serie de Tiempo, } \\
\text { establecimiento, estimación } \\
\text { directa de función de costos o } \\
\text { de producción, otros países }\end{array}$ & $\begin{array}{r}\text { Horas-trabajador operativas } \\
\text { manufactureras, trimestralmente, } \\
\text { 1956-78; Reino Unido; condición } \\
\text { de producto marginal, sistemas } \\
\text { oferta-demanda }\end{array}$ & $\begin{array}{l}{[0.49,} \\
0.79]\end{array}$ \\
\hline Lewis y Kirby (1988) & $\begin{array}{l}\text { Serie de Tiempo, } \\
\text { establecimiento, estimación } \\
\text { directa de función de costos o } \\
\text { de producción, otros países. }\end{array}$ & $\begin{array}{r}\text { Empleo no agrícola, } \\
\text { trimestralmente, 1967-87; } \\
\text { Australia; condición de producto } \\
\text { marginal }\end{array}$ & 0.78 \\
\hline Rudebusch (1986) & $\begin{array}{l}\text { Serie de Tiempo, } \\
\text { establecimiento, estimación } \\
\text { directa de función de costos o } \\
\text { de producción, E.U. }\end{array}$ & $\begin{array}{r}\text { Horas-trabajador de negocios no } \\
\text { agrícolas, trimestralmente, } 1952-81 ; \\
\text { condición de producto marginal, } \\
\text { modelo oferta-demanda de } \\
\text { desequilibrio }\end{array}$ & 1.16 \\
\hline Quandt y Rosen (1988) & $\begin{array}{l}\text { Serie de Tiempo, } \\
\text { establecimiento, estimación } \\
\text { directa de función de costos o } \\
\text { de producción, E.U. }\end{array}$ & $\begin{array}{r}\text { Horas-trabajador privado, anual, } \\
\text { 1932-83, condición de producto } \\
\text { marginal, modelo oferta-demanda } \\
\text { de desequilibrio }\end{array}$ & 0.69 \\
\hline $\begin{array}{lc}\text { Hall, } & \text { Henry } \\
\text { Pemberton (1990) }\end{array}$ & $\begin{array}{l}\text { Serie de Tiempo, } \\
\text { establecimiento, estimación } \\
\text { directa de función de costos o } \\
\text { de producción, otros páses }\end{array}$ & $\begin{array}{l}\text { Empleo total, trimestralmente, } \\
\text { 1966-88; Reino Unido; modelo } \\
\text { oferta-demanda de desequilibrio }\end{array}$ & 1.73 \\
\hline $\begin{array}{llll}\text { Brown } & \text { y } & \text { de } & \text { Cani } \\
(1963) & & & \end{array}$ & $\begin{array}{l}\text { Serie de Tiempo, } \\
\text { establecimiento, estimación } \\
\text { directa de función de costos o } \\
\text { de producción, E.U. }\end{array}$ & $\begin{array}{r}\text { Horas-trabajador privadas no } \\
\text { agrícolas, anual, 1933-58; razón } \\
\text { capital-trabajo }\end{array}$ & 0.47 \\
\hline $\begin{array}{l}\text { David } \quad \mathrm{y} \quad \text { van } \\
\text { Klundert (1965) }\end{array}$ & $\begin{array}{l}\text { Serie de Tiempo, } \\
\text { establecimiento, estimación } \\
\text { directa de función de costos o } \\
\text { de producción, E.U. }\end{array}$ & $\begin{array}{l}\text { Horas-trabajador privadas, anual, } \\
\text { 1899-1960; relación capital-trabajo }\end{array}$ & 0.32 \\
\hline Schaafsma (1978) & $\begin{array}{l}\text { Serie de Tiempo, } \\
\text { establecimiento, estimación } \\
\text { directa de función de costos o } \\
\text { de producción, otros países }\end{array}$ & $\begin{array}{r}\text { Empleo manufacturero, anual, } \\
\text { 1949-72; Canadá; razón capital- } \\
\text { trabajo }\end{array}$ & 0.42 \\
\hline Symons (1985) & $\begin{array}{l}\text { Serie de Tiempo, } \\
\text { establecimiento, estimación } \\
\text { directa de función de costos o } \\
\text { de producción otros países }\end{array}$ & $\begin{array}{r}\text { Empleo manufacturero, } \\
\text { trimestralmente, 1961-76; Reino } \\
\text { Unido; KL y precios de } \\
\text { importaciones }\end{array}$ & 2.40 \\
\hline
\end{tabular}

Nota: los valores presentados entre corchetes, por ejemplo [0.49, 0.79$]$, indican el rango de valores entre los que se encuentra la elasticidad de sustitución $\sigma_{\mathrm{LK}}$ estimada, que en este caso se encuentra entre un mínimo de 0.49 y un

Fuente: Hamermesh, 1993, cuadro 3.1: 78-79.

Revista de Economía - Vol. XXVIII - Núm. 78

\section{Cuadro 2. Estimaciones de $\sigma$ utilizando datos de pequeñas industrias}

\begin{tabular}{|c|c|c|c|}
\hline Estudio & Categoría & Descripción & \\
\hline McKinnon (1962) & $\begin{array}{l}\text { Series de Tiempo, } \\
\text { establecimiento, estimación } \\
\text { directa de función de costos o } \\
\text { de producción, E.U. }\end{array}$ & $\begin{array}{r}\text { Clases manufactureras a dos dígitos, } \\
\text { anuales, 1947-58; condición de } \\
\text { producto marginal }\end{array}$ & 0.29 \\
\hline Lovell (1973) & $\begin{array}{l}\text { Corte Transversal, } \\
\text { establecimiento, estimación } \\
\text { directa de función de costos o } \\
\text { de producción, E.U. }\end{array}$ & $\begin{array}{l}\text { Clases manufactureras, empelo } \\
\text { producción-trabajador, estados, } \\
\text { 1958, función de producción de } \\
\text { elasticidad de sustitución variable. }\end{array}$ & 0.75 \\
\hline $\begin{array}{ll}\text { Ashenfelter } & \text { and } \\
\text { Ehrenberg (197) }\end{array}$ & $\begin{array}{l}\text { Datos panel, establecimiento, } \\
\text { estimación directa de función de } \\
\text { costos o de producción, E.U.. }\end{array}$ & $\begin{array}{l}\text { Actividades de gobiernos estatales y } \\
\text { locales, estados, } 1958-69 \text {; condición } \\
\text { de producto marginal. }\end{array}$ & 0.67 \\
\hline Freeman (1975) & $\begin{array}{l}\text { Serie de Tiempo, } \\
\text { establecimiento, estimación } \\
\text { directa de función de costos o } \\
\text { de producción, E.U. }\end{array}$ & $\begin{array}{r}\text { Facultad de la universidad, anual, } \\
\text { 1920-70; condición de producto } \\
\text { marginal. }\end{array}$ & 0.26 \\
\hline Thornton (1979) & $\begin{array}{l}\text { Corte Transversal, } \\
\text { establecimiento, estimación } \\
\text { directa de función de costos o } \\
\text { de producción, E.U. }\end{array}$ & $\begin{array}{l}\text { Maestros de escuelas públicas, } \\
\text { estados, } 1968-74 ; \text { condición de } \\
\text { producto marginal. }\end{array}$ & 0.63 \\
\hline Bernanke (1986) & $\begin{array}{l}\text { Serie de Tiempo, } \\
\text { establecimiento, estimación } \\
\text { directa de función de costos o } \\
\text { de producción, E.U, }\end{array}$ & $\begin{array}{r}\text { Pequeñas industrias manufactureras, } \\
\text { empleo producción-trabajador, } \\
\text { mensualmente, 1923-39; condición } \\
\text { de producto marginal, oferta- } \\
\text { demanda. }\end{array}$ & 0.58 \\
\hline Stapleton (1989) & $\begin{array}{l}\text { Serie de Tiempo, } \\
\text { establecimiento, estimación } \\
\text { directa de función de costo o de } \\
\text { producción, E.U. }\end{array}$ & $\begin{array}{r}\text { Economistas Ph. D., anual, 1960-85; } \\
\text { condición de producto marginal; } \\
\text { sistema oferta-demanda con } \\
\text { especificación de expectativas de los } \\
\text { oferentes. }\end{array}$ & 0.06 \\
\hline
\end{tabular}

En ambos cuadros podemos observar que todas las estimaciones de $\sigma \mathrm{LK}$ presentadas tienen valor positivo denotando la condición de sustituibilidad de dichos factores; es decir, que cuando sube el precio de uno de ellos sube también la demanda del otro factor y viceversa. Asimismo, observamos que el rango de valores entre los que se encuentra dicha elasticidad para datos agregados o de grandes industrias es de entre 0.21 y 2.40 , con promedio de 0.80 y mediana de 0.68 ; mientras que las elasticidades estimadas para datos de industrias pequeñas se encuentra en un rango de valores de entre 0.06 y 0.75 , con promedio de 0.46 y mediana de 0.58 , lo cual implica que la sustitución entre los factores trabajo y capital es mayor 
para las industrias más grandes o para datos agregados que para datos de industrias pequeñas o para datos desagregados.

Como complemento de los cuadros 1 y 2 y con el fin de presentar estimaciones más recientes de la elasticidad de sustitución capital-trabajo, mostramos en el cuadro 3 estimaciones realizadas en estudios que distintos autores han llevado a cabo de 1993 a la fecha de tal elasticidad, en las cuales podemos observar que en su gran mayoría de casos se reportan valores positivos, corroborando los resultados presentados en los cuadros 1 y 2 , en los que casi todos las elasticidades estimadas son positivas, indicando que son sustitutos ambos factores productivos. Otro resultado que podemos advertir en el cuadro 3 es que la mayoría de las elasticidades de sustitución estimadas por los autores es inferior a 1, lo cual indica que la sustitución entre el trabajo y el capital es relativamente baja. La descripción de las columnas del cuadro 3 es la misma que para los cuadros 1 y 2 .

Cuadro 3.

Estimaciones recientes de $\boldsymbol{\sigma}$

\begin{tabular}{|c|c|c|c|}
\hline Estudio & Categoría: & Descripción: & $\sigma_{\mathrm{LK}}$ \\
\hline Chirinko (1993) & $\begin{array}{l}\text { Series de Tiempo, establecimiento, estimación } \\
\text { directa de modelos de inversión EU. }\end{array}$ & $\begin{array}{r}\text { Datos trimestrales del sector manufacturero, precios } \\
\text { canidadads shocks } 1970-1990 .\end{array}$ & $0.10 \mathrm{a} 0.30$ \\
\hline Clark (1993) & $\begin{array}{l}\text { Datos panel, establecimientio, estimación directa de } \\
\text { función producción Cobb-Douglas con } \\
\text { expectativas estáicas EU. }\end{array}$ & 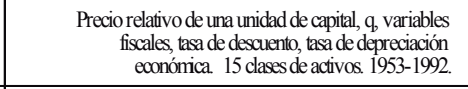 & $0.18 \mathrm{a} 0.28$ \\
\hline $\begin{array}{l}\text { Caballero etal. } \\
\text { (1995) }\end{array}$ & 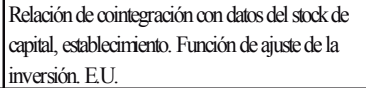 & 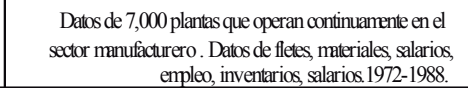 & 0.70 \\
\hline $\begin{array}{l}\text { Cummins, Hassety } \\
\text { Hubbard (1994) }\end{array}$ & 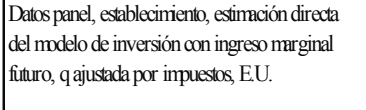 & 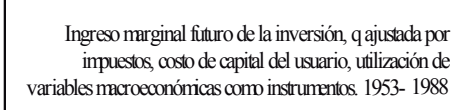 & 0.67 \\
\hline $\begin{array}{l}\text { Chirinko, Fazzari y } \\
\text { Meyer (2002) }\end{array}$ & $\begin{array}{l}\text { Datass panel, extablecimiento, stimmión directa de } \\
\text { un modelo de ciclo de negocios, EU. }\end{array}$ & 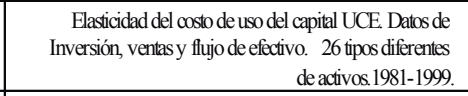 & $0.30 \mathrm{a} 0.50$ \\
\hline $\begin{array}{l}\text { Jorgenson y Young } \\
\text { (2001) }\end{array}$ & $\begin{array}{l}\text { Modelo de costo de capital basado en una finción } \\
\text { producción, EU. Familias empressan no } \\
\text { hucraivas empressas lucrativas }\end{array}$ & 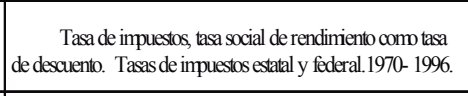 & $\begin{array}{l}\text { Lucrativas } 0.50, \\
\text { No lucrativa, } \\
0.70\end{array}$ \\
\hline Jabbar Saed (2002) & $\begin{array}{l}\text { Series de Tiempo. Funcíón de produccóín CES } \\
\text { de Arrow, Chenery, Minhasy Solow. } \\
\text { Establecimiento. Minimos caadradosordinarios. } \\
\text { Jordania. }\end{array}$ & 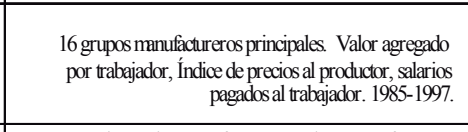 & -0.068 al. .05 \\
\hline Juselius (2005) & 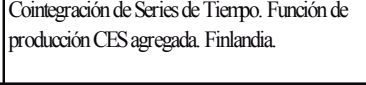 & 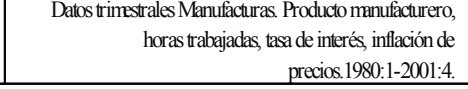 & 1.39 \\
\hline
\end{tabular}

Revista de Economía - Vol. XXVIII - Núm. 78

\begin{tabular}{|c|c|c|c|}
\hline Sirapattanakul (2005) & $\begin{array}{l}\text { Función producoción CES con rendimientios } \\
\text { constantes a escalla Correa }\end{array}$ & 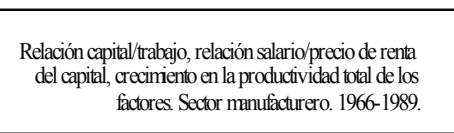 & $\begin{array}{l}0.62 \mathrm{a} 2.18 \mathrm{en} \\
1970-1979 ; 0.87 \\
\text { a } 1.29 \mathrm{en} 1980- \\
1989\end{array}$ \\
\hline $\begin{array}{l}\text { Upender y Sujan } \\
\text { (2009) }\end{array}$ & 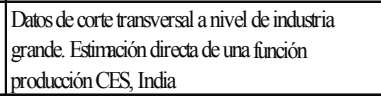 & 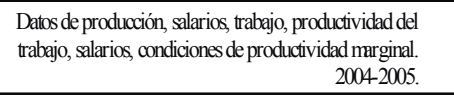 & 1.2835 \\
\hline Young (2010) & 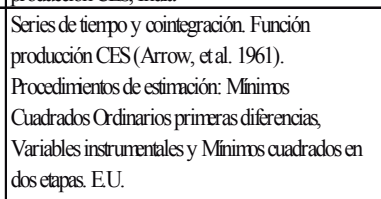 & 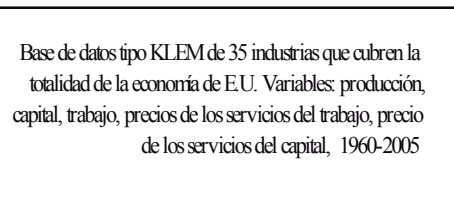 & $0.526 \mathrm{a} 0.547$ \\
\hline Chirinko (2008) & \begin{tabular}{|l|} 
Función producción CES con oostos de ajuste. \\
Ora versión es obtenida mediante los costos del \\
ussuario de capitall. EU. y ortos países
\end{tabular} & 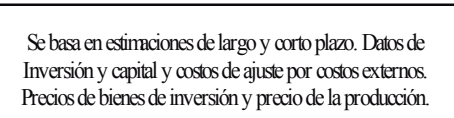 & $0.4 \mathrm{a} 0.6$ \\
\hline Okagawa (2008) & 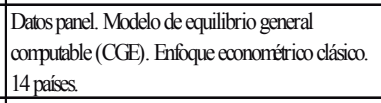 & $\begin{array}{l}\text { Datos del proyecto KLEM- EU, } 19 \text { industrias. Estimn } \\
\text { elasticidades en las estructuras de produciocion KE-Ly KL- } \\
\text { E } 1980-1990 .\end{array}$ & 0.85 a 1.26 \\
\hline $\begin{array}{l}\text { Raurich, Salay } \\
\text { Sorolla (2011) }\end{array}$ & 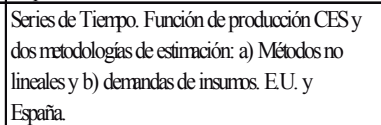 & 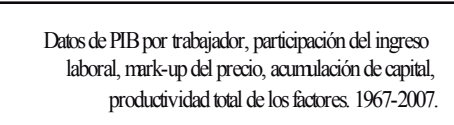 & \begin{tabular}{|l} 
España 1.58; \\
EU. 0.51
\end{tabular} \\
\hline
\end{tabular}

Mención especial dentro de la literatura existente sobre el tema merece el estudio denominado KLEM debido a los símbolos de los factores de producción en el utilizados, el capital K, el trabajo L, los energéticos E y los materiales M, realizado por Ernst Berndt utilizando las especificaciones Translogarítmica y Leontief de la demanda de trabajo y datos de la industria manufacturera de Estados Unidos a nivel de firma para el año de 1971 y el procedimiento de estimación del "estimador iterativo eficiente de Zellner," cuyos resultados son presentados en el cuadro 4, referentes a las elasticidades precio propias y cruzadas de los cuatro factores utilizados, así como las elasticidades de sustitución entre los mismos. (Berndt, 1991: 464-465, 475-476), (Hamermesh, 1996: 101) 
Cuadro 4.

Elasticidades precio y de sustitución de la demanda de trabajo (KLEM)

\begin{tabular}{|c|c|c|c|c|c|}
\hline \multicolumn{3}{|c|}{ Elasticidades de Sustitución } & \multicolumn{3}{|c|}{$\begin{array}{l}\text { Elasticidades precio } \\
\end{array}$} \\
\hline$\sigma_{i j}$ & \begin{tabular}{|l|} 
Leontief \\
Generalizada
\end{tabular} & Translog & $\eta_{i j}$ & $\begin{array}{l}\text { Leontief } \\
\text { Generalizada }\end{array}$ & Translog \\
\hline$\sigma_{\mathrm{KL}}$ & 6.84 & 0.97 & $\eta_{\mathrm{KL}}$ & 0.62 & 0.29 \\
\hline$\sigma_{\mathrm{KE}}$ & -1.98 & -3.60 & $\eta_{\mathrm{KE}}$ & -0.05 & -0.16 \\
\hline$\sigma_{\mathrm{KM}}$ & -0.87 & 0.35 & $\eta_{K M}$ & -0.28 & 0.21 \\
\hline$\sigma_{\mathrm{LM}}$ & 1.04 & 0.61 & $\eta_{\mathrm{KK}}$ & -0.29 & -0.34 \\
\hline$\sigma_{\mathrm{EM}}$ & 0.15 & 0.83 & $\eta_{\mathrm{LK}}$ & 0.11 & 0.05 \\
\hline$\sigma_{\mathrm{LE}}$ & 11.14 & 0.68 & $\eta_{\mathrm{LE}}$ & 0.13 & 0.03 \\
\hline$\sigma_{\mathrm{KK}}$ & -5.50 & -6.99 & $\eta_{\mathrm{LM}}$ & 0.14 & 0.37 \\
\hline$\sigma_{\mathrm{LL}}$ & -1.27 & -1.51 & $\eta_{\mathrm{LL}}$ & -0.38 & -0.45 \\
\hline$\sigma_{\mathrm{EE}}$ & -13.72 & -11.74 & $\eta_{\mathrm{EK}}$ & -0.05 & -0.17 \\
\hline$\sigma_{\mathrm{MM}}$ & $\begin{array}{r}-0.09 \\
\end{array}$ & -0.39 & $\eta_{\mathrm{EL}}$ & 0.74 & 0.20 \\
\hline & \multicolumn{2}{|c|}{ Elasticidades Sustituc ión } & \multicolumn{3}{|c|}{ Elasticidades precio } \\
\hline & & & $\prod_{\text {㤑 }}$ & -0.72 & -0.53 \\
\hline & & & $\eta_{\mathrm{MK}}$ & -0.02 & 0.02 \\
\hline & & & $\eta_{\mathrm{ML}}$ & 0.07 & 0.18 \\
\hline & & & $\eta_{\mathrm{ME}}$ & 0.003 & 0.04 \\
\hline & & & $\eta_{\text {мM }}$ & -0.05 & -0.24 \\
\hline \multicolumn{3}{|c|}{ 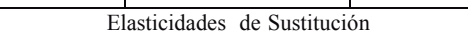 } & \multicolumn{3}{|c|}{ Elasticidades precio } \\
\hline$\sigma_{i j}$ & \begin{tabular}{|l|l|} 
Leontief \\
Generalizada
\end{tabular} & Translog & $\eta_{i j}$ & $\begin{array}{l}\text { Leontief } \\
\text { Generalizada }\end{array}$ & Translog \\
\hline$\sigma_{\mathrm{KL}}$ & 6.84 & 0.97 & $\eta_{\mathrm{KL}}$ & 0.62 & 0.29 \\
\hline$\sigma_{\mathrm{KE}}$ & -1.98 & -3.60 & $\eta_{\mathrm{KE}}$ & -0.05 & -0.16 \\
\hline$\sigma_{\mathrm{KM}}$ & -0.87 & 0.35 & $\eta_{\mathrm{KM}}$ & -0.28 & 0.21 \\
\hline$\sigma_{\mathrm{LM}}$ & 1.04 & 0.61 & $\eta_{\mathrm{KK}}$ & -0.29 & -0.34 \\
\hline$\sigma_{\mathrm{EM}}$ & 0.15 & 0.83 & $\eta_{\mathrm{LK}}$ & 0.11 & 0.05 \\
\hline$\sigma_{\mathrm{LE}}$ & 11.14 & 0.68 & $\eta_{\mathrm{LE}}$ & 0.13 & 0.03 \\
\hline$\sigma_{\mathrm{KK}}$ & -5.50 & -6.99 & $\eta_{\mathrm{LM}}$ & 0.14 & 0.37 \\
\hline$\sigma_{\mathrm{LL}}$ & -1.27 & -1.51 & $\eta_{\mathrm{LL}}$ & -0.38 & -0.45 \\
\hline$\sigma_{\mathrm{EE}}$ & -13.72 & -11.74 & $\eta_{\mathrm{EK}}$ & -0.05 & -0.17 \\
\hline \multirow[t]{6}{*}{$\sigma_{\mathrm{MM}}$} & $\begin{array}{r}-0.09 \\
\end{array}$ & -0.39 & $\eta_{\mathrm{EL}}$ & 0.74 & 0.20 \\
\hline & Elasticidades Sustituc i & & $\eta_{\mathrm{EM}}$ & \multicolumn{2}{|c|}{ Elasticidades preci } \\
\hline & & & $\eta_{\mathrm{EE}}$ & -0.72 & -0.53 \\
\hline & & & $\eta_{\mathrm{MK}}$ & -0.02 & 0.02 \\
\hline & & & $\eta_{\mathrm{ML}}$ & 0.07 & 0.18 \\
\hline & & & $\eta_{\mathrm{ME}}$ & 0.003 & 0.04 \\
\hline
\end{tabular}

Fuente: Elaboración propia, con base en (Berndt, 1991: 464-465, 475-476).

Revista de Economía - Vol. XXVIII - Núm. 78
Como podemos notar en el cuadro 4, la elasticidad de sustitución entre los factores productivos trabajo $\mathrm{L}$ y capital $\mathrm{K}$ es positiva denotando que ambos son sustitutos, asimismo que el valor de esa elasticidad es de 0.97 para la Translogarítmica, lo que indica un grado de sustitución relativamente bajo entre estos factores debido al bajo nivel de agregación de los datos. También podemos inferir de dicho cuadro que las elasticidades precio de la demanda de trabajo y de la demanda de capital son menores a la unidad, denotando que ambos factores presentan una demanda inelástica, siendo más inelástica la de capital. Asimismo, que las elasticidades precio cruzadas entre ambos factores es positiva, indicando que son sustitutos el trabajo y el capital.

\section{METODOLOGÍA DE LA INVESTIGACIÓN}

3.1 Descripción de datos y medición de variables:

Para este estudio se tiene información de un total de 282 clases que componen el sector manufacturero mexicano para los dos años correspondientes a los Censos Industriales (1999 y 2004), y que agrupan a las empresas de cada clase. En estos censos se captó información de todos los establecimientos 15 del país que durante las etapas de levantamiento censal (del 1 de marzo al 30 de junio de 1999 y del 1 de marzo al 30 de junio de 2004 respectivamente) estaban desarrollando alguna

15"Establecimiento es la unidad económica asentada en una sola ubicación física, de manera permanente y delimitada por construcciones e instalaciones fijas, que combina acciones y recursos bajo el control de una sola entidad propietaria o controladora, para realizar actividades de producción de bienes, maquila total o parcial de uno o varios productos, la compra-venta de mercancías o prestación de servicios, sea con fines .

Enero a Junio de 2012 - Págs: 37-79 
actividad económica no primaria 16, del país que durante las etapas de levantamiento censal (del 1 de marzo al 30 de junio de 1999 y del 1 de Marzo al 30 de junio de 1999 y del 1 de marzo al 30 de junio de 2004 respectivamente) estaban desarrollando alguna actividad económica no primaria, en el apartado correspondiente a los censos sobre manufacturas, por ejemplo, elaboración de productos de todo tipo: alimenticios, metálicos, químicos, etc., incluyendo maquila. (INEGI 2004:1) Es decir, que los datos se refieren exclusivamente a la industria manufacturera, dejando de lado datos correspondientes a otras actividades económicas del sector secundario (por ejemplo transporte), así como a todas aquellas del sector primario y del terciario.

Los datos utilizados presentan la mayor desagregación posible, que es a nivel de clase de actividad, en virtud de que INEGI no tiene disponible información a nivel de unidad económica. Esta desagregación económica está de acuerdo con los niveles que componen la clasificación usada por el Sistema de Clasificación Industrial de América del Norte (SCIAN), clasificador de actividades económicas oficiales del INEGI desde 1997 en estos censos. Para el estudio de la economía mexicana, el SCIAN la divide en 20 sectores, 95 subsectores, 309 ramas, 631 subramas y, en su nivel más detallado, en 1051 clases de actividad. De estas últimas, los Censos Económicos 2004 hicieron la cobertura de 971 17. (INEGI 2004:1) En los censos industriales, se incluye información de 282 clases, las cuales contienen información de 328,718 unidades económicas.

A su vez, las clases se agrupan en 182 subramas, mismas que se integran en 86 ramas, las cuales están constituidas en 21 subsectores, correspondientes al sector manufacturero.

${ }^{6}$ El estudio de las actividades del sector primario de la economía pertenece al Censo Agropecuario; en los Censos Económicos 2004, de estas actividades sólo fueron objeto de censo la pesca y la acuicultura. Otros unidades extraterritoriales (como embajadas, consulados, unidades militares), además de los partidos políticos y otras organizaciones políticas (ligas, coaliciones, frentes de lucha, etc.) INEGI, 2004: 2

${ }^{17} \mathrm{El}$ número de las clases de actividad cubiertas por los censos económicos, particularmente los industriales, es menor al total de clases existentes, debido a que en las zonas urbanas se lleva a cabo el censo, no así en las zonas rurales, en las que se realiza un muestreo; asimismo tampoco se incluyen las unidades económicas del sector público o de organizaciones religiosas, ni las que iniciaron sus actividades en el año del censo INEGI, 2004: 3

$$
\text { Revista de Economía - Vol. XXVIII - Núm. } 78
$$

Aunque los censos económicos están diseñados para generar información de corte transversal, es decir, información sobre un momento del tiempo, por ejemplo, para comparar una zona geográfica contra otra o una actividad económica contra otra actividad económica; es también factible comparar directamente los resultados de los censos del 2004 con los resultados definitivos de los Censos Económicos de 1999. (INEGI 2004:7) 18 (INEGI 2003), (INEGI 2005) Por lo anterior pudo construirse una combinación de cortes transversales independientes con dos censos (1999 y 2004) para 282 clases, que son datos de corte transversal 19 .

Las variables que forman parte de las ecuaciones estimadas se enlistan en el cuadro 5 y se describen en los párrafos siguientes:

Cuadro 5:

Nomenclatura de las variables utilizadas en los modelos econométricos

\begin{tabular}{|c|c|}
\hline $\mathrm{L}_{1}$ & $\begin{array}{l}\text { Número de trabajadores operativos y administrativos (con derecho a prestaciones } \\
\text { extra salariales ). }\end{array}$ \\
\hline $\mathrm{L}_{2}$ & $\begin{array}{l}\text { Número de trabajadores por honorarios o comisiones (sin derecho a prestaciones } \\
\text { extra salariales ). }\end{array}$ \\
\hline $\mathrm{K}$ & Cantidad de capital utilizado por las empresas. \\
\hline $\mathrm{w}_{1}$ & $\begin{array}{l}\text { Salario pagado a cada trabajador operativo o administrativo (con prestaciones extra } \\
\text { salariales ) }\end{array}$ \\
\hline $\mathrm{w}_{2}$ & Honorarios o comisiones pagadas por trabajador (sin prestaciones extra salariales) \\
\hline $\mathrm{w}_{\mathrm{K}}$ & Precio del capital \\
\hline $\mathrm{Y}$ & Valor de la producción de las empresas \\
\hline $\mathrm{C}$ & $\begin{array}{l}\text { Costo total (costo total de salarios sumado al costo total de prestaciones, las } \\
\text { indemnizaciones por despido el monto del reparto de utilidades, el costo de } \\
\text { adquisición de capital y el costo de energéticos) }\end{array}$ \\
\hline
\end{tabular}

18 "La comparación es posible en virtud de que los resultados de los censos economicos de 1999 fueron dados a conocer, por única vez y para facilitar a los usuarios el paso hacia la nueva clasificación desde la optica de dos clasificadores. El Sistema de Clasificación Industrial de América del Norte (SCIAN), el clasificador de actividades economicas oficial del INEGI desde 1997, y la Clasificación Mexicana de Actividades y Productos (CMAP) de 1994, clasificación que venía empleándose desde 1981". (INEGI 2004:7), (INEGI 1997), (INEGI 2004).

19Por única ocasión en 2004 los resultados del Censo 1999 fueron convertidos a la versión del SCIAN utilizada en el Censo 2004, que es la versión 2002, para facilitar su comparación. Sin embargo, los resultados del Censo Económico 2009 son también comparables con los del Censo 2004 ya que en el Anexo de éste se muestran las modificaciones que se hicieron al SCIAN del Censo de 2009 respecto al SCIAN del Censo

Enero a Junio de 2012 - Págs: 37-79 
El número de trabajadores con prestaciones laborales (L1), se calculó con base en los trabajadores que recibieron salarios y sueldos pagados así como prestaciones extra salariales (prestaciones sociales, reparto de utilidades y costos de despido). Esta variable incluye a los obreros, es decir, a las personas que realizaron trabajos ligados con la operación de la maquinaria en la fabricación de bienes, supervisores de línea, así como el personal vinculado a tareas auxiliares en el proceso de producción dedicado a la provisión de materias primas, embalaje, despacho, almacenaje, mantenimiento y limpieza de la planta, transporte, etc,; a los que se sumaron los trabajadores administrativos o empleados administrativos, es decir, las personas que desempeñaron labores de oficina, administración, contabilidad, actividades auxiliares y complementarias; así como labores ejecutivas, de planeación, organización, dirección y control. (INEGI 2004: 8). El número de trabajadores sin prestaciones laborales (L2), se calculó con base en los trabajadores que no recibieron prestaciones extra salariales (prestaciones sociales, reparto de utilidades ni costos de despido), e incluyó la cantidad de personal por honorarios o comisiones sin sueldo base, reportada en los censos económicos, es decir, los trabajadores que laboraron por cuenta propia para el establecimiento y que cobraron exclusivamente con base en honorarios, comisiones o igualas. Considera a las personas que recibieron como pago exclusivamente propinas, pero excluye a abogados, médicos, contadores y demás profesionistas que cobraron honorarios o igualas por la prestación de sus servicios profesionales, sin laborar de manera regular o exclusiva para el establecimiento. (INEGI 2004:10). Las remuneraciones recibidas por este grupo se denominan w2 en este trabajo.

Para medir la cantidad de capital demandada por las empresas (K), se utilizaron las compras de activos fijos, (reportadas en los censos económicos), es decir, el monto de las adquisiciones y transferencias recibidas de activos fijos a valor actual, nuevos o usados, que durante el período de referencia realizó el establecimiento, sumando todas las erogaciones en que incurrió para llevar e instalar los activos fijos en la unidad económica.
Esta cifra incluye las mejoras, reformas y renovaciones de importancia que realizó el establecimiento en sus activos fijos para incrementar la productividad o vida útil de éstos y la producción de activos fijos para uso propio pero excluye las reparaciones y mantenimiento corriente de los activos fijos. A lo anterior se agregó la depreciación de activos fijos, que comprende la pérdida de valor durante el año de referencia por el uso u obsolescencia de los activos fijos propiedad del establecimiento. (INEGI 2004:28). Esta medida sirve entonces de proxy (variable aproximada) para el uso del capital en un año dado.

Para medir los salarios pagados a los trabajadores con previsiones laborales (w1), se utilizó la nómina de salarios y sueldos pagados a obreros por los establecimientos para retribuir el trabajo ordinario y extraordinario de este tipo de personal dependiente de la razón social antes de cualquier deducción retenida por los empleadores (impuesto sobre la renta o sobre el producto del trabajo), las aportaciones de los trabajadores a los regímenes de seguridad social (IMSS, ISSSTE, INFONAVIT) y las cuotas sindicales (reportados en los censos económicos). Esta cifra incluye aguinaldos; comisiones sobre ventas que complementan el sueldo base; primas vacacionales; bonificaciones, incentivos y bonos de productividad pero a su vez excluye los gastos en pasajes y viáticos, alimentos y en general los gastos reembolsados al trabajador. (INEGI 2004:12). Lo anterior se sumó a las prestaciones laborales (independientes de los sueldos y salarios) que enseguida se describen.

a) el total de prestaciones sociales, que incluye por un lado las contribuciones patronales a regímenes de seguridad social, es decir, los pagos que realiza la unidad económica a favor de su personal, a las instituciones de seguridad social (IMSS, ISSSTE, INFONAVIT, etcétera), así como las aportaciones de fondos de ahorro para el retiro, y por otro lado los pagos contractuales y extracontractuales que proporciona la unidad económica a los trabajadores como una remuneración adicional a los sueldos y salarios, ya sea en dinero o en especie, tales como: servicio médico privado, despensas, primas de seguros al personal, servicios educativos, ayudas para estudio y guarderías;

Enero a Junio de 2012 - Págs: 37-79 
b) las utilidades repartidas a los trabajadores, es decir, los pagos en efectivo que la unidad económica distribuye entre el personal antes de cualquier deducción, por concepto de utilidades conforme a la legislación laboral o las cláusulas de los contratos de trabajo o convenios entre patrones y trabajadores, cualquiera que sea el ejercicio al que correspondan; y c) pagos por indemnización o liquidación al personal, que se refiere a la compensación monetaria que se otorga a los trabajadores por concepto de despido, retiro o terminación de contrato laboral con la unidad económica. (INEGI 2004:14).

La suma de los conceptos anteriores se dividió entre el número de operarios y empleados administrativos (L1), es decir, w1 representa el costo laboral promedio en trabajadores con previsiones laborales, tomando en consideración los costos por prestaciones sociales, utilidades repartidas y los costos de despido.

Para medir los honorarios o comisiones pagados a los trabajadores que laboraron bajo esta modalidad (w2), se utilizaron los honorarios o comisiones reportados en los censos económicos, que son el importe de los pagos que realizaron los establecimientos por este concepto al personal que no recibe una remuneración base. Esta cifra incorpora el pago por concepto de igualas pero excluye las comisiones pagadas en adición a un salario base.

(INEGI 2004:14). Lo anterior se dividió entre el número de trabajadores por honorarios o comisiones sin sueldo base, es decir, w2 representa el costo laboral promedio en trabajadores sin previsiones laborales.
El precio de renta del capital (wK), se determinó con base en el promedio diario de la TIIE (tasa de interés interbancaria de equilibrio) a 28 días 20 , utilizando el promedio diario durante los años de 1998 y 2003. (www.banxico.org.mx).

La totalidad de los datos utilizados en las regresiones fueron convertidos a términos reales, a pesos de 2004, utilizando el Índice Nacional de Precios al Consumidor (INPC) que tuvo un valor promedio de 81.9344 en la segunda quincena de junio de 1999 y de 109.69 en la segunda quincena de junio de 2004 (www.banxico.org.mx). Las bases de datos en términos nominales y reales se reportan en los cuadros 6 y 7 respectivamente.

Cuadro 6:

Descripción de variables en términos nominales por clase industrial

\begin{tabular}{|c|c|c|c|c|c|}
\hline & & AÑO & MÍNIMO & PROMEDIO & MÁXIMO \\
\hline $\mathrm{w}_{1}$ & $\begin{array}{l}\text { SALARII DE UN TRABAJADOR } \\
\text { OPERATIVO (miles de pesos) }\end{array}$ & $\begin{array}{l}1999 \\
2004\end{array}$ & $\begin{array}{r}6.43 \\
21.26\end{array}$ & $\begin{array}{l}27.47 \\
49.48\end{array}$ & $\begin{array}{l}106.46 \\
230.30\end{array}$ \\
\hline $\mathrm{w}_{2}$ & $\begin{array}{l}\text { SALARIO DE UN TRABAJADOR } \\
\text { ADMINISTRATIVO (miles de } \\
\text { pesos) }\end{array}$ & $\begin{array}{l}1999 \\
2004\end{array}$ & $\begin{array}{r}8.54 \\
45.36\end{array}$ & $\begin{array}{r}75.83 \\
132.18\end{array}$ & $\begin{array}{l}252.08 \\
742.25\end{array}$ \\
\hline $\mathrm{w}_{3}$ & $\begin{array}{l}\text { SALARIO DE UN TRABAJADOR } \\
\text { POR HONORARIOS (miles de } \\
\text { pesos) }\end{array}$ & $\begin{array}{l}1999 \\
2004\end{array}$ & $\begin{array}{l}0.00 \\
0.00\end{array}$ & $\begin{array}{l}502.35 \\
112.25\end{array}$ & $\begin{array}{l}7091.30 \\
4345.05\end{array}$ \\
\hline $\mathrm{w}_{4}$ & $\begin{array}{l}\text { PRESTACIONES SOCIALES, } \\
\text { REPARTO DE UTILIDADES Y } \\
\text { COSTOS DE } \\
\text { DESPIDO POR } \\
\text { TRABAJADOR (miles } \\
\text { de pesos) }\end{array}$ & 1999 & 0.25 & 16.96 & 80.33 \\
\hline $\mathrm{w}_{\mathrm{K}}$ & $\begin{array}{l}\text { PECIO DEL } \\
\text { CAPITAL (\% } \\
\text { anual) }\end{array}$ & $\begin{array}{l}1999 \\
2004\end{array}$ & $\begin{array}{r}18.23 \\
4.97\end{array}$ & $\begin{array}{r}23.99 \\
7.17 \\
\end{array}$ & $\begin{array}{r}40.01 \\
9.11\end{array}$ \\
\hline
\end{tabular}

${ }^{20}$ Como alternativa también se utilizó la tasa de retorno de la inversión, (en este caso el cociente entre las utilidades repartidas a los trabajadores y el stock de capital de las empresas) como Proxy del precio del capital, sin embargo, debido a que al correrse las regresiones utilizando dicha Proxy se obtuvieron algunos resultados de elasticidades que están fuera del rango reportado en la literatura empírica sobre el tema, no se incluyen en este trabajo.

Enero a Junio de 2012 - Págs: 37-79 
$\begin{array}{ll} & \\ & \text { PRECIO DE LOS } \\ \text { ENERGÉTICOS (pesos) }\end{array}$

\begin{tabular}{|c|c|c|c|c|}
\hline 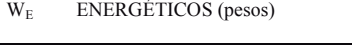 & 2004 & & $\begin{array}{r}91.81 \\
100\end{array}$ & \\
\hline $\begin{array}{l}\text { No. DE } \\
\text { TRABAJADORES } \\
\text { OPERATIVOS }\end{array}$ & $\begin{array}{l}1999 \\
2004\end{array}$ & $\begin{array}{r}4 \\
12\end{array}$ & $\begin{array}{r}10,197 \\
9,629 \\
\end{array}$ & $\begin{array}{l}245,800 \\
216,840\end{array}$ \\
\hline $\begin{array}{l}\text { No. DE } \\
\text { TRABAADRRES } \\
\text { ADMINISTRATIVOS }\end{array}$ & 1999 & 9 & $\begin{array}{l}2,756 \\
2.075\end{array}$ & $\begin{array}{l}28,626 \\
38,054\end{array}$ \\
\hline $\begin{array}{l}\text { No. DE } \\
\text { TRBAAADORES POR } \\
\text { HONORARIOS } \\
\end{array}$ & $\begin{array}{l}1999 \\
2004\end{array}$ & $\begin{array}{l}0 \\
0\end{array}$ & $\begin{array}{l}184 \\
129\end{array}$ & $\begin{array}{l}2,708 \\
2,071 \\
\end{array}$ \\
\hline $\begin{array}{l}\text { CAPTTAL } \\
\text { DEMANDADO (miles } \\
\text { de pesos) }\end{array}$ & $\begin{array}{l}1999 \\
2004\end{array}$ & $\begin{array}{r}5.00 \\
13.00\end{array}$ & $\begin{array}{l}423,516.30 \\
659,233.00\end{array}$ & $\begin{array}{r}3,773,970.00 \\
13,000,000.00\end{array}$ \\
\hline $\begin{array}{l}\text { ENERGÉTICOS } \\
\text { CONSMMIDOS (miles } \\
\text { de pesos) }\end{array}$ & $\begin{array}{l}1999 \\
2004\end{array}$ & $\begin{array}{r}154.00 \\
37.00\end{array}$ & $\begin{array}{r}\begin{array}{l}172,423.70 \\
272,150.80\end{array} \\
\end{array}$ & $\begin{array}{r}4,467,659.00 \\
10,100,000.00 \\
\end{array}$ \\
\hline $\begin{array}{l}\text { COSTOS } \\
\text { TOTALES } \\
\text { (miles de pesos) }\end{array}$ & $\begin{array}{l}1999 \\
2004 \\
\end{array}$ & $\begin{array}{l}323.00 \\
832.00\end{array}$ & $\begin{array}{l}1,133,785.00 \\
1,949,050.00\end{array}$ & $\begin{array}{l}\begin{array}{l}13,200,000.00 \\
34,700,000.00\end{array} \\
\end{array}$ \\
\hline $\begin{array}{l}\text { VALOR DE LOS PRODUCTOS } \\
\text { ELABORADOS (miles de pesos) }\end{array}$ & $\begin{array}{l}1999 \\
2004\end{array}$ & $\begin{array}{l}0.00 \\
0.00\end{array}$ & $\begin{array}{l}5,181,860.00 \\
5,181,860.00\end{array}$ & $\begin{array}{r}75,500,000.00 \\
130,000,000.00\end{array}$ \\
\hline $\begin{array}{l}\text { COSTOS DE DESPIDO } \\
\text { (miles de pesos) }\end{array}$ & $\begin{array}{l}1999 \\
2004\end{array}$ & $\begin{array}{l}0.00 \\
0.00\end{array}$ & $\begin{array}{r}8,773.80 \\
59,870.20\end{array}$ & $\begin{array}{r}146,219.00 \\
9,434,687.00 \\
\end{array}$ \\
\hline $\begin{array}{l}\text { TOTAL DE PREETACIONES } \\
\text { SOCIALES (miles de pesos) }\end{array}$ & $\begin{array}{l}1999 \\
2004\end{array}$ & $\begin{array}{r}211.00 \\
55.00\end{array}$ & $\begin{array}{l}167,148.00 \\
218,011.30\end{array}$ & $\begin{array}{l}2,883,551.00 \\
4,477.280 .00\end{array}$ \\
\hline $\begin{array}{l}\text { OTRAS } \\
\text { PRESTACIONES } \\
\text { SOCCALES (miles de } \\
\text { pesos) }\end{array}$ & $\begin{array}{l}1999 \\
2004\end{array}$ & $\begin{array}{l}3.00 \\
0.00\end{array}$ & $\begin{array}{r}96,575.04 \\
184,691.00\end{array}$ & $\begin{array}{l}2,883,551.00 \\
1,987,606.00\end{array}$ \\
\hline $\begin{array}{l}\text { REPARTO DE } \\
\text { UTIIIDDDES } 99 \text { (miles } \\
\text { de pesos) } \\
\text { REPARTO DE } \\
\text { UTIIIDADES } 04 \text { (miles } \\
\text { de pesos) }\end{array}$ & 1999 & 0.00 & $29,715.03$ & $1,036,694.00$ \\
\hline
\end{tabular}

Notas:

/Se determinó dividiendo la nómina total por el número de trabajadores

2/ Se determinó dividiendo el total de estos conceptos por el total de trabajadores operativos y administrativos

Se determinó utilizando el como precio del capital el promedio diario de la TIIE a 28 días durante Le Se determinó promedi

promediando los precios de las gasolinas diesel, magna y premium, así como de la $5 /$ Se determino

capital ocurridas en ese año. 6/ Se determinaron suman

7/ Incluye aportaciones patronales a los fondos para el retiro

FUENTE: Censos Económicos 1999 y 2004. INEGI.

Revista de Economía - Vol. XXVIII - Núm. 78
Cuadro 7:

Descripción de variables en términos reales por clase industrial (pesos de 2004)

\begin{tabular}{|c|c|c|c|c|c|}
\hline & & ANO & MINIMO & PROMEDIO & MÁXIMO \\
\hline $\mathrm{w}_{\mathrm{i}}$ & 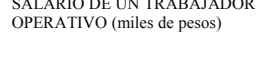 & $\begin{array}{l}1999 \\
2004\end{array}$ & $\begin{array}{r}8.61 \\
2.126\end{array}$ & $\begin{array}{l}36.77 \\
44.48\end{array}$ & $\begin{array}{l}142.53 \\
220.30\end{array}$ \\
\hline $\mathrm{w}_{2}$ & 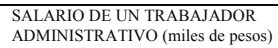 & 1999 & 11.43 & 101.52 & $\begin{array}{l}250.30 \\
337.50\end{array}$ \\
\hline & & 2004 & 45.36 & 132.18 & 742.25 \\
\hline $\mathrm{w}_{3}$ & 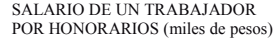 & 1999 & 0.00 & 672.57 & \\
\hline & & 2004 & 0.00 & 112.25 & $4,345.05$ \\
\hline $\mathrm{w}_{4}$ & 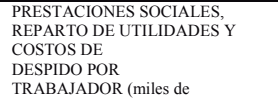 & 1999 & 0.33 & 22.70 & \\
\hline & 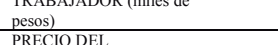 & 2004 & 2.16 & 29.48 & $1,097.78$ \\
\hline $\mathrm{w}_{\mathrm{k}}$ & $\begin{array}{l}\text { CAPITAL } \% \\
\text { anual) }\end{array}$ & 1999 & 18.23 & 23.99 & 40.01 \\
\hline & & 2004 & 4.97 & 7.17 & \\
\hline $\mathrm{w}_{\mathrm{E}}$ & ENERGÉTICOS (pesos) & $\begin{array}{r}1999 \\
2004 \\
\end{array}$ & & $\begin{array}{r}122.91 \\
100\end{array}$ & \\
\hline $\mathrm{L}_{1}$ & $\begin{array}{l}\text { NoD DE TRABAADDORES } \\
\text { OPERATIVOS }\end{array}$ & 1999 & 4 & 10,197 & 245,800 \\
\hline & & 2004 & 12 & 9,629 & 216,840 \\
\hline $\mathrm{L}_{2}$ & 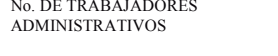 & 1999 & 9 & 2,756 & 28,626 \\
\hline $\mathrm{L}_{3}$ & $\begin{array}{l}\text { No. DE TRABAADOORES } \\
\text { POR HONORARIOS }\end{array}$ & 1999 & 0 & 184 & 2,708 \\
\hline & CAPITAL DEMANDADO & 2004 & & 129 & \\
\hline к & (miles de pessos) & 1999 & 6.69 & $567,032.13$ & $5,052,845.09$ \\
\hline E & $\begin{array}{l}\text { ENERGËTIICOS } \\
\text { CONNUMMIDOS (miles de } \\
\text { pessos) }\end{array}$ & $\begin{array}{l}1999 \\
2004\end{array}$ & $\begin{array}{r}206.18 \\
3700\end{array}$ & $\begin{array}{l}230,852.45 \\
272,150.80 \\
27\end{array}$ & $\begin{array}{l}5,981,602.62 \\
1.1000000\end{array}$ \\
\hline c & $\begin{array}{l}\text { CoSTOS } \\
\text { TOTALS (miles } \\
\text { de pesos) }\end{array}$ & $\begin{array}{r}1999 \\
2004 \\
\end{array}$ & $\begin{array}{l}432.45 \\
832.00\end{array}$ & $\begin{array}{l}1,517,987.68 \\
1,949,050.00\end{array}$ & $\begin{array}{l}17,699,395,30 \\
34,700,000.00\end{array}$ \\
\hline Y & 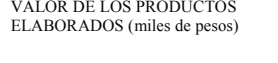 & $\begin{array}{l}1999 \\
2004\end{array}$ & 0.00 & $\begin{array}{l}6,937,823.00 \\
51818000\end{array}$ & ${ }_{101,084,482.50}^{100000}$ \\
\hline & $\begin{array}{l}\text { COSTros DE EESPIDO } \\
\text { (miles de pesos) }\end{array}$ & 1999 & 0.00 & $\begin{array}{l}1.181,800.00 \\
11,746.95\end{array}$ & $195,767.84$ \\
\hline & & & 0.00 & $59,870.20$ & 9.434 .687 .00 \\
\hline & 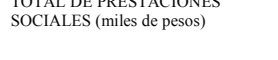 & $\begin{array}{l}1999 \\
2004\end{array}$ & $\begin{array}{r}282.50 \\
59.00\end{array}$ & $\begin{array}{l}223,788.99 \\
218.011 .30\end{array}$ & $\begin{array}{l}3,860,692.19 \\
4.473 .286 .00\end{array}$ \\
\hline & 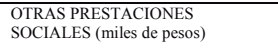 & 1999 & 4.02 & 129,30 & $3,860,692.19$ \\
\hline & & & & $\begin{array}{l}184,691.00 \\
\end{array}$ & $1.987,606.4$ \\
\hline & 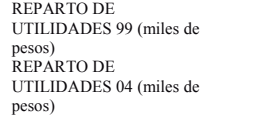 & $\begin{array}{l}1999 \\
2004\end{array}$ & 0.00 & $39,784,48$ & \\
\hline
\end{tabular}

Nota: Los valores a precios de 2004 se obtuvieron utilizando los valores del INPC de 1999 y 2004, que fueron 81.93 y 109.69.

el cuadro anterio.

Enero a Junio de 2012 - Págs: 37-79 


\section{ANÁLISIS DE RESULTADOS}

\subsection{Ecuaciones estimadas}

El sistema de ecuaciones simultáneas estimadas se basa en la especificación Translogarítmica similar a la del modelo KLEM, pero compuesta de 3 ecuaciones correspondientes a las demandas de los siguientes factores de las empresas manufactureras: trabajo Formal (L1), que comprende a todos los trabajadores que cuentan con prestaciones extra salariales, es decir a los trabajadores operativos y administrativos; trabajo Informal (L2), que comprende a los trabajadores que no cuentan con prestaciones extra salariales, es decir a los trabajadores por honorarios y comisiones, y Capital $(\mathrm{K})$, que comprende las adquisiciones de capital y la depreciación de capital de las empresas.

Las demandas de los factores son representadas por las participaciones de los costos de los factores en los costos totales, con los nombres S1, S2 y SK respectivamente. Los precios de estos tres factores son w1, w2 y wK respectivamente e incluyen, el primero el salario y las prestaciones extrasalariales (prestaciones sociales, reparto de utilidades y costos de despido) recibidos por los trabajadores de planta de la empresa (obreros y administrativos), el segundo sólo los honorarios y comisiones recibidas, y el tercero la tasa de interés interbancaria de equilibrio (TIIE).

$\mathrm{S}_{1}=\mathrm{a}_{1}+\mathrm{b}_{11} \ln \mathrm{w}_{1}+\mathrm{b}_{12} \ln \mathrm{w}_{2}+\mathrm{b}_{1 \mathrm{~K}} \ln \mathrm{w}_{\mathrm{K}}+\mathrm{b}_{1 \mathrm{Y}} \ln \mathrm{Y}$

(9) $\quad \mathrm{S}_{2}=\mathrm{a}_{2}+\mathrm{b}_{21} \operatorname{lnw_{1}}+\mathrm{b}_{22} \operatorname{lnw}_{2}+\mathrm{b}_{2 \mathrm{~K}} \ln \mathrm{w}_{\mathrm{K}}+\mathrm{b}_{2 \mathrm{Y}} \ln \mathrm{Y}$

$\mathrm{S}_{\mathrm{K}}=\mathrm{a}_{\mathrm{K}}+\mathrm{b}_{\mathrm{K} 1} \operatorname{lnw}_{1}+\mathrm{b}_{\mathrm{K} 2} \operatorname{lnw}_{2}+\mathrm{b}_{\mathrm{KK}} \ln \mathrm{ln}_{\mathrm{K}}+\mathrm{b}_{\mathrm{KY}} \ln \mathrm{Y}$
Realizamos la estimación de cada una de estas ecuaciones mediante el procedimiento de cortes transversales independientes o estimación pooled utilizando el procedimiento de mínimos cuadrados ordinarios, ecuación por ecuación, en el cual suponemos que el error en cada ecuación del sistema no está correlacionado con la variables explicativas en cada una de las ecuaciones, es decir que no hay problemas de endogeneidad $2_{21}$ en cada una de las ecuaciones que constituyen el sistema porque los precios son variables exógenas; sin embargo, si existiese correlación entre los errores y las variables explicativas porque se cree que los precios son variables endógenas, la estimación de mínimos cuadrados ordinarios produce estimadores inconsistentes y se requiere adoptar otros métodos de estimación, y el problema puede ser enfrentado mediante mínimos cuadrados en dos etapas (si se cuenta con variables instrumentales idóneas) o con métodos para datos panel (si se cuenta con datos de este tipo).

En el cuadro 8 se reportan los resultados correspondientes a las estimaciones para la función de costos translogarítmica de este sistema de ecuaciones bajo el procedimiento de mínimos cuadrados ordinarios con datos combinando los dos cortes transversales sin separarlos según el año de censo.

Cuadro 8. Resultados econométricos para el sistema de ecuaciones Translogarítmico Pooled

\begin{tabular}{|l|c|}
\hline \multicolumn{1}{|c|}{ Variable } & Coeficientes MCO Pooled \\
\hline Determinantes de la variable $w_{1} L_{I} / C$ ó participación del trabajo con prestaciones sociales en los costos \\
totales
\end{tabular}

21-En el contexto de sistemas de ecuaciones simultáneas se denominan variables endógenas aquellas que son conjuntamente dependientes, es decir, que no es posible estimar los parámetros de una ecuación aisladamente, sin tener en cuenta la información proporcionada por las demás ecuaciones del sistema, por lo que hay más de una ecuación del sistema, una para cada una de las variables conjuntamente dependientes. (Gujarati, 2004:

Enero a Junio de 2012 - Págs: 37-79 
Cuadro 8.

Resultados econométricos para el sistema de ecuaciones Translogarítmico Pooled

\begin{tabular}{|c|c|}
\hline Salario de trabajadores con prestaciones $\left(\mathrm{W}_{1}\right)$ & -0.033 \\
\hline Salario de trabajadores sin prestaciones $\left(\mathrm{W}_{2}\right)$ & $\frac{(-2.1)}{-0.0208}$ \\
\hline & $(-4.477 * *$ \\
\hline Precio del capital (r) & 0.052 \\
\hline & $(4.43)^{* *}$ \\
\hline Valor de la producción $(\mathrm{Y})$ & -0.02 \\
\hline $\mathrm{R}^{2}$ & $\frac{(-5.66)^{* * *}}{0.21}$ \\
\hline Determinantes de la variable $w_{2} L_{2} / C$ ó partici & restaciones sociales en los cost \\
\hline Variable & Coeficientes MCO Pooled \\
\hline Constante & 0.11 \\
\hline & $(0.59)$ \\
\hline Salario de trabajadores con prestaciones $\left(\mathrm{W}_{1}\right)$ & -0.013 \\
\hline & $(-4.27)^{* *}$ \\
\hline Salario de trabajadores sin prestaciones $\left(\mathrm{W}_{2}\right)$ & 0.015 \\
\hline Precio del capital (r) & $\frac{(13.63)^{* * *}}{0.013}$ \\
\hline & $(4.65)^{* *}$ \\
\hline Valor de la producción $(\mathrm{Y})$ & -0.002 \\
\hline & $\frac{(-2.76)}{0.36}$ \\
\hline 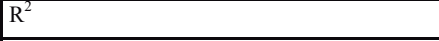 & 0.36 \\
\hline Determinantes de la variable $w_{K} K / C$ & ital en los costos totales \\
\hline \begin{tabular}{|l|l|} 
Constante \\
\end{tabular} & -0.31 \\
\hline & $(-4.71)$ \\
\hline Salario de trabajadores con prestaciones $\left(\mathrm{W}_{1}\right)$ & 0.046 \\
\hline & $(3.86)^{* *}$ \\
\hline Salario de trabajadores sin prestaciones $\left(\mathrm{W}_{2}\right)$ & 0.004 \\
\hline & (1.08) \\
\hline Precio del capital (r) & 0.038 \\
\hline & $(3.34)^{* *}$ \\
\hline Valor de la producción $(\mathrm{Y})$ & 0.023 \\
\hline & $(6.50)^{* *}$ \\
\hline $\mathrm{R}^{2}$ & 0.17 \\
\hline
\end{tabular}

Los valores entre paréntesis muestran el estadístico t correspondiente a cada uno de los coeficientes estimados.**

Fuente: Elaboración propia con base en datos de los Censos Económicos 1999 y 2004. INEGI.
Los resultados permiten apreciar la considerable significancia estadística de los coeficientes estimados, lo cual confirma de alguna manera la exogeneidad de las variables independientes ya que cada una de las ecuaciones del sistema conservan su independencia respecto al resto de ellas, a pesar de ser parte del sistema. Cabe mencionar también el impacto positivo y significativo del capital sobre la demanda del trabajo con prestaciones L1 y sobre la demanda de trabajo sin prestaciones L2, es decir, que son sustitutos K y L1 asimismo K y L2. También se observa el impacto negativo y significativo entre ambos tipos de trabajo, lo que sugiere cierta complementariedad entre el trabajo con prestaciones extra salariales y el trabajo sin prestaciones extra salariales.

La estimación de las elasticidades precio propias y cruzadas de las demandas de factores así como las elasticidades de sustitución entre factores se presentan en el cuadro 8. La forma en que se calcularon dichas elasticidades fue mediante la aplicación de las fórmulas (5) a (8), utilizando los coeficientes de las regresiones translogarítmicas así como los valores promedio de todas las observaciones de precios de los factores, todas las cantidades utilizadas de los factores, los costos y el valor de la producción. Los errores estándar de las estimaciones de las elasticidades precio de la demanda se calcularon mediante el paquete econométrico Stata, simultáneamente con el cálculo de las elasticidades precio de las demandas de factores, aplicando el comando correspondiente a post-estimación. Los resultados obtenidos muestran un valor de 1.00 para la elasticidad propia del trabajo formal (el que cuenta con prestaciones), de 0.39 para la elasticidad propia del trabajo por honorarios (que no cuenta con prestaciones) y de 0.39 para la elasticidad cruzada del trabajo sin prestaciones respecto al trabajo con prestaciones. Por ello, por cada punto porcentual en que se redujeran las prestaciones, se esperaría un aumento de uno por ciento en la demanda de trabajo formal, y de 0.39 por ciento en la demanda de trabajo por honorarios. 
Cuadro 9.

Elasticidades precio y elasticidades de sustitución de la demanda de factores productivos

\begin{tabular}{|l|c|r|r|r|r|r|}
\hline & \multicolumn{2}{|c|}{$\mathrm{L}_{1}$} & \multicolumn{2}{c|}{$\mathrm{L}_{2}$} & \multicolumn{2}{c|}{$\mathrm{K}$} \\
\hline & $\sigma_{\mathrm{ij}}$ & \multicolumn{1}{c|}{$\eta_{\mathrm{ij}}$} & $\sigma_{\mathrm{ij}}$ & \multicolumn{1}{c|}{$\eta_{\mathrm{ij}}$} & $\sigma_{\mathrm{ij}}$ & \multicolumn{1}{c|}{$\eta_{\mathrm{ij}}$} \\
\hline $\mathrm{w}_{1}$ & -0.61 & $-1.00 *$ & -0.41 & $-0.39 * *$ & 1.28 & $-1.57^{* *}$ \\
& & $(0.02)$ & & $(0.09)$ & & $(0.36)$ \\
\hline $\mathrm{w}_{2}$ & -0.29 & $-0.03 * *$ & -15.26 & $-0.39 * *$ & 1.31 & 0.00 \\
& & $(0.00)$ & & $(0.05)$ & & $(0.00)$ \\
\hline $\mathrm{w}_{\mathrm{K}}$ & 0.67 & $-0.07 * *$ & 2.04 & 0.00 & -1.52 & -0.00 \\
& & $(0.01)$ & & $(0.00)$ & & $(0.00)$ \\
\hline
\end{tabular}

Nota: los valores entre paréntesis muestran los errores estándar de las elasticidades precio estimadas. El símbolo ** representa una significancia estadística de $95 \%$ de las elasticidades precio estimada. Fuente: Cruz, M., 2011, Cuadro Anexo 2: 242.

En cuanto a los resultados de la elasticidad de sustitución entre el capital y el trabajo se observan valores positivos de 1.28 y 1.31 del trabajo con prestaciones y el trabajo sin prestaciones respectivamente respecto al capital, lo que implica sustituibilidad de ambos tipos de trabajo con el capital, por lo que una disminución en el precio de cualquiera de los dos tipos de trabajo conduciría a un aumento en la utilización de trabajo y a una menor utilización de capital, con lo que se refuerza la hipótesis de que son sustitutos el trabajo y el capital en la industria manufacturera mexicana. También se observa que la elasticidad de sustitución estimada es mayor a 1, por lo que es alta la sustituibilidad entre ambos tipos de trabajo y el capital. Asimismo, se advierte que la elasticidad de sustitución entre ambos tipos de trabajo es negativa, por lo que el trabajo con prestaciones extra salariales es complementario del trabajo sin prestaciones.

La evidencia empírica encontrada para el caso mexicano, comparada con la evidencia empírica encontrada en otros países nos muestra que es superior la sustitución entre el trabajo y el capital en nuestro país, mostrando que las empresas mexicanas son más propensas a sustituir capital por trabajo que en otros países, sea este último con prestaciones o sin presta- ciones, en la medida en que el precio relativo de alguno de estos factores aumenta, utilizando más capital cuando el precio del trabajo aumenta, y utilizando más trabajo cada vez que el precio del factor capital aumenta. Los anterior nos conduce a conjeturar que la productividad marginal de los factores productivos considerados (trabajo con prestaciones, trabajo sin prestaciones y capital) es mayor en México que en otros países, lo que ocasionaría que la sensibilidad de los mercados de estos factores a cambios en los precios de los otros factores sea más alta en nuestro país.

\section{CONCLUSIONES}

Este trabajo muestra la segmentación que existe en el mercado de trabajo del sector manufacturero mexicano en un sector formal que es aproximado por el trabajo con prestaciones extrasalariales, y un sector informal que es aproximado por el trabajo sin prestaciones extrasalariales, así como el impacto que tienen los cambios en los remuneraciones de los trabajadores de dichos sectores sobre la demanda de trabajo tanto con prestaciones como sin prestaciones.. Asimismo, el impacto que tienen los cambios en las remuneraciones de los trabajadores sobre la utilización del capital y el grado de sustituibilidad entre el trabajo y el capital.

Como podemos deducir de las estimaciones de las elasticidades de sustitución presentadas en el cuadro 9, tanto el trabajo con prestaciones extrasalariales como el trabajo sin prestaciones extrasalariales son sustitutos de manera significativa respecto del factor capital, por lo que cualquier reducción en el salario de alguno de los dos tipos de trabajo ocasionaría un aumento en la demanda de trabajo y una reducción en la contratación de capital, asimismo, un aumento en alguno de los dos tipos de salario conduciría al aumento de capital contratado. 
No obstante, es necesario recalcar el alto valor de la elasticidad de sustitución estimada para la industria manufacturera mexicana entre el trabajo con prestaciones y el capital (1.28) así como entre el trabajo sin prestaciones y el capital (1.31), por lo que no observamos una diferencia significativa en el grado de sustitución entre el trabajo y el capital ocasionado por la existencia de los costos extrasalariales, ya que por igual el trabajo con prestaciones extra salariales y el trabajo que no cuenta con ellas es sustituto de manera significativa con el capital.

Con lo anterior damos respuesta a la pregunta de investigación formulada al inicio de nuestro trabajo, respondiendo que entre los factores trabajo y capital en la industria manufacturera mexicana si existe sustituibilidad y ésta es más alta que en otros países, por lo que sugerimos un mayor cuidado en las políticas públicas dirigidas a cambiar los precios relativos de dichos factores, porque se estarían ocasionando impactos considerables en los mercados de los mismos. Asimismo, que no se debe la alta sustituibilidad entre el trabajo y el capital a la presencia de costos extrasalariales, sino más bien a la alta productividad marginal de estos factores, lo que los hace altamente sensibles a los cambios en los precios relativos de los mismos.

Por otra parte, los resultados de las estimaciones mostradas en el cuadro 8 muestran además un valor de 1.00 para la elasticidad propia del trabajo formal (el que cuenta con prestaciones), de 0.39 para la elasticidad propia del trabajo por honorarios (que no cuenta con prestaciones) y de 0.39 para la elasticidad cruzada del trabajo sin prestaciones respecto al trabajo con prestaciones. Por ello, por cada punto porcentual en que se redujeran las prestaciones, se esperaría un aumento de uno por ciento en la demanda de trabajo formal, y de 0.39 por ciento en la demanda de trabajo por honorarios.

Acerca de las limitaciones de este estudio, cabe destacar la insuficiencia del análisis que por simplificación considera como proxy del trabajo formal el trabajo con prestaciones extra salariales y como trabajo informal el trabajo sin prestaciones extra salariales, lo que limita la representatividad de nuestra muestra, ya que sería necesario considerar muchos factores cualitativos y cuantitativos más para definir adecuadamente a los sectores laborales formal e informal. Asimismo, hacemos notar que nuestro estudio utiliza el procedimiento de estimación econométrica de cortes transversales independientes o pooled, suponiendo que no existe el problema de la endogeneidad en las ecuaciones del sistema, lo cual es otra limitación, ya que no podemos descartar el problema de las variables endógenas y utilizar para su corrección el procedimiento de variables instrumentales cuando se tienen suficientes instrumentos o el de datos panel cuando se tienen datos de este último tipo. 


\section{REFERENCIAS:}

Behrman, Jere H. (1982). "Country and Sectoral Variations in Manufactu ring Elasticities of Substitution between Capital and Labor", NBER, vol. 2, pp. 159-192.

Berndt, Ernst, y David Wood (1975). "Technology, Prices, and the Derived Demand of Energy." Review of Economics and Statistics, $57: 25968$

Berndt, Ernst (1991). The practice of Econometrics classic and contempo rary. Addison-Wesley Publishing Company. United States.

Banco Interamericano de Desarrollo (2010). La era de la productividad: cómo transformar la economía desde sus cimientos. BID.

Boon, Gerardo K. (1973). “Sustitución de capital y trabajo, comparaciones de productividad e insumos primarios proyectado",Demografía y Economía, el Colegio de México, Vol. VII, No. 3.

Caballero, Ricardo, et al. (1995). "Plant-Level Adjustment and Aggregate Investment Dynamics", Brookings Papers on Economic Activity, Vol. 1995, Issue 2, 1-54.

Cahuc, Pierre y André Zylberberg (2004). Labour Economics. Cambridge, Massachussets: The MIT Press.

Clark, Peter K. (1993). "Tax Incentives and Equipment Investment", Brookings Papers on Economic Activity, Vol. 1993, Issue 1, 317 347.Claro, Sebastian (2002). "A Cross Country Estimation of the Elasticity of Substitution between Labor and Capital in Manufacturing Industries", Cuadernos de Economía, Universidad Católica de Chile, Vol.40, No. 120, pp. 239-2.57.

Chirinko, Robert S. (1993). "Business Fuxed Investment Spending: Modeling Strategies, Empirical Results and Policy Implications", Journal of Economic Literature, Vol. 31, No. 4, December, 18751911.

Chirinko, Robert S., Steven M. Fazzari y Andrew P. Meyer (2002). "That Elusive Elasticity: A long-panel Approach to Estimation of Business Capital", mimeo, CESIFO Working Paper Series No. 1240, January.
Chirinko, Robert S. (2008). "б. The Long and Short of It", CESIFO Working Paper No. 2234, Category 1: Public Finance, February 2008.

Cruz, Miguel (2011). El impacto de los costos de despido sobre la demanda de trabajo con referencia al caso de la industria manufacturera mexicana, Universidad José Vasconcelos, Oaxaca, México.

Cummins, Jason G., Kevin A. Hasset y R. Glenn Hubbard (1994). “A Reconsideration of Investment Behavior Using Tax Reforms as Natural Experiments", Brooking Papers on Economic Activity, 2: $1-72$.

Gujarati, Damodar, (2005). Econometría, Mc Graw Hill, México Hamermesh, Daniel S. (1993). Labor Demand, Princeton: Princeton University Press.

Ibarra, Jorge (1994). "Price Elasticities and Substitution Elasticities Among Productive Factors in the Border Mexican Maquiladora Industry", Serie Documentos de Trabajo del Departamento de Economía del ITESM, Noviembre.

Instituto Nacional de Estadística, Geografía e Informática (2001). Metodología de los Censos Económicos 1999.

Instituto Nacional de Estadística, Geografía e Informática (2003). Censos Económicos 1999. Sistema Automatizado de Información Censal SAIC 4.0 SCIAN y CMAP. (discos compactos)

Instituto Nacional de Estadística, Geografía e Informática (2005). Censos Económicos 2004. Sistema Automatizado de Información Censal SAIC 5.0. (disco compacto)

Instituto Nacional de Estadística, Geografía e Informática (2004). Metodología de los Censos Económicos 2004.

Instituto Nacional de Estadística, Geografía e Informática (2004). Cuestionario para la Industria Manufacturera. XVI Censo Industrial, www.inegi.org.mx

Instituto Nacional de Estadística, Geografía e Informática (2009). Metodología de los Censos Económicos 2009, Anexo B.

Jabbar Saed Alaf A. (2002) "Technological Progress and CapitalLabour Substitution in the Jordany Industry: 1985-1997”, Journal of Economics \& Administrative Sciences, Vol. 18, No. 2, December. 
Jirapattanakul, Pimchanok (2005). "Elasticity of Substitution Evidence of Assimilation in Korea", a thesis submitted in partial fullfillment of the requirement for the Degree of Bachelor of Arts in Economics, William College, Williamstown, Massachusetts, Mayo 23.

Jorgenson, Dale W. y Kun-Young Yun (2001). Investment, Volume 3.Lifting the Burden: Tax Reform, The Cost of Capital, and U.S. Economic Growth, The MIT Press, Cambridge, Massachusetts 02142.

Juselius, Mikael (2005). "Log-Run Relationships Between Labor and Capital: Indirect Evidence on the Elasticity of Sustitution", Discussion Paper No. 57, Abril, Helsinky Center of Economic Research.

Lora, Eduardo y Carmen Pagés-Serra (1997). La Legislación Laboral en el proceso de reformas estructurales de América Latina y el Caribe, Banco Interamericano de Desarrollo-Oficina del Economista en Jefe, Diciembre.

Lora, Eduardo (2001). Las Reformas estructurales en América Latina: Qué se ha reformado y cómo medirlo, RES Working Papers 4288, Inter-American Development Bank, Research Department, December.

Montes Rojas, Gabriel, y Mauricio Santamaría (2007). "The burden of labor costs in Mexico". Labour 21(1) 157-188

Nicholson, Walter (1997). Teoría Microeconómica. Madrid: Editorial Mc Graw Hill Interamericana.

Okagawa, Azusa y Kanemi Ban (2008). "Estimation of Substitution Elasticities for CGE models", Discussion Papers in Economics and Business, Osaka University, Graduate School of Economics and Osaka School of International Public Policy, number 08-16, Abril.

Parkin,Michael, Gerardo Esquivel y Marcos Ávalos (2005). Microeco nomía. Versión para Latinpoamérica, Pearson Addison Wesley, séptima edición, México.
Raurich, Xavier, Héctor Sala y Valeri Sorolla (2011). "Factor Shares, the Price Markup and the Elasticity of Substitution between capital and labor", Discussion Paper Series, IZA DP No. 59992, Forschunginstitut zur Zukunt der Arbeit Institute for the study of labor, Septiembre.

The World Bank, the International Finance Corporation, and Oxford University. (2004). Doingbusiness in 2004. Undestanding Regulation, Copublication of the World Bank and Oxford University Press, Washington, D.C., www.worldbank.org

Upender, Mulakala y M. Sujan (2009) "Differential Elasticity of Substi tution in the IndianIndustries", Romanian Journal of Economic Forecasting, Volumen 3, 47-55.

Wooldridge, Jeffrey M. (2001). "Introducción a la Econometría, un enfoque moderno. México: Thomson Learning.

Young, Andrew T. (2010). "U.S: Elasticities of Substitution and Factor Augmentation at the Industry Level", Working Paper Series, College of Business and Economics, Division of Economics and Finance, West Virginia University.

\section{Referencias electrónicas}

www.eumed.net/c; www.oecd.org/dataoecd/48/32/45633680.pdf http://www.eumed.net/cursecon/ecolat/mx/2005/mcro-informal.htm Banco de México. Indicadores Económicos

www.banxico.org.mx 